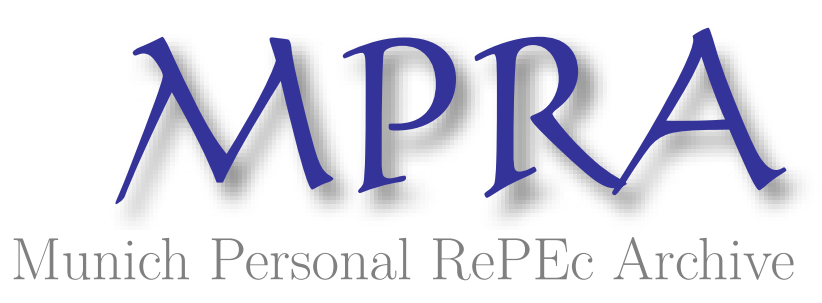

\title{
Estimation of Log-GARCH Models in the Presence of Zero Returns
}

Sucarrat, Genaro and Escribano, Alvaro

BI Norwegian Business School, Universidad Carlos III de Madrid

9 September 2013

Online at https://mpra.ub.uni-muenchen.de/75010/

MPRA Paper No. 75010, posted 11 Nov 2016 12:46 UTC 


\title{
Estimation of Log-GARCH Models in the Presence of Zero Returns $^{1}$
}

\author{
Genaro Sucarrat ${ }^{2}$ and Alvaro Escribano ${ }^{3}$ \\ First version: 9 September 2013 \\ This version: 3 October 2016
}

\begin{abstract}
A critique that has been directed towards the $\log$-GARCH model is that its log-volatility specification does not exist in the presence of zero returns. A common "remedy" is to replace the zeros with a small (in the absolute sense) non-zero value. However, this renders estimation asymptotically biased. Here, we propose a solution to the case where the true return is equal to zero with probability zero. In this case zero returns may be observed because of non-trading, measurement error (e.g. due to rounding), missing values and other data issues. The algorithm we propose treats the zeros as missing values and handles these by estimation via the ARMA representation. An extensive number of simulations verify the conjectured asymptotic properties of the bias-correcting algorithm, and several empirical applications illustrate that it can make a substantial difference in practice.
\end{abstract}

JEL Classification: C22, C58

Keywords: ARCH, exponential GARCH, log-GARCH, ARMA, inliers, missing values

1 Introduction 2

2 Model, framework and algorithm 4

2.1 The log-GARCH model .............. 4

2.2 Observed zeros - a framework ........... 6

2.3 Algorithm ................. 8

3 A Monte Carlo study 10

3.1 Effect of zeros . . . . . . . . . . . . . . 10

3.2 Properties of algorithm . . . . . . . . . . . 11

3.3 Can we condition on estimated zero-probabilities? . . . . 12

4 Empirical applications $\quad 13$

4.1 The effect of zero-returns on volatility . . . . . . . 13

4.2 Apple: Return volatility, zeros and volume . . . . . 15

$\begin{array}{lll}5 & \text { Conclusions } & 17\end{array}$

References $\quad 19$

${ }^{1}$ We are grateful to the Editor and reviewers for their helpful comments, suggestions and questions, and to participants at the CFE 2014 (Pisa), NSVCM 2014 (Paderborn) and ISNPS 2014 (Cadiz) conferences. Alvaro Escribano acknowledges financial support from the Spanish Ministry of Economy and Competitiveness ECO2015-68715-R, ECO2016-00105-001, Consolidation Grant \#2006/04046/002 and Maria de Maeztu Grant MDM 2014-0431.

${ }^{2}$ Corresponding author. Department of Economics, BI Norwegian Business School, Nydalsveien 37, 0484 Oslo, Norway. Email genaro.sucarrat at bi.no, phone $+47+46410779$, fax $+47+23264788$.

${ }^{3}$ Department of Economics, Universidad Carlos III de Madrid (Spain), email: alvaroe at eco.uc3m.es. 


\section{Introduction}

Models in the Autoregressive Conditional Heteroscedasticity (ARCH) class due to Engle (1982) have been extensively used to model the time-varying volatility of financial return (see Francq and Zakoïan (2010) for a survey of ARCH models). In particular, the firstorder Generalised ARCH model of Bollerslev (1986), i.e. the GARCH(1,1), has become especially popular, since it parsimoniously captures the volatility persistence commonly exhibited by financial returns. Pantula (1986), Geweke (1986) and Milhøj (1987) independently proposed specifications within the log-ARCH class of models as an alternative to non-exponential ARCH models. Their main motivation was to ensure the positivity of fitted volatilities - this is not guaranteed in non-exponential ARCH models (in particular when additional exogenous or predetermined conditioning information is added), and to allow for richer dynamics (e.g. negative ARCH parameters for cyclical or contrarian dynamics). ${ }^{4}$ Engle and Bollerslev (1986) argued against log- $\mathrm{ARCH}$ models because of the possibility of applying the log-operator on zero-values. ${ }^{5}$ This occurs whenever the return or mean-corrected return equals zero. Subsequently Nelson (1991) proposed an alternative exponential ARCH specification, the EGARCH model, where the problem is sidestepped by replacing the problematic term with an expression that does not involve the log operator. This solution, however, comes at a considerable cost: Restrictive assumptions and complicated conditions are needed to ensure that the Quasi Maximum Likelihood Estimator (QMLE) provides Consistent and Asymptotically Normal (CAN) estimates (Wintenberger (2013)), and unconditional moments (e.g. the unconditional variance of returns) will generally not exist for $t$-distributed densities (see condition (A1.6) and the subsequent discussion in Nelson (1991, p. 365)).

Zero returns occur in two different types of situations. In the first the zero-probability of actual return is zero, but zeros are nevertheless observed due to, say, non-trading, discreteness approximation error (e.g. rounding error), missing values and other data issues. For example, missing quotes or transaction prices are typically replaced by the previous observation, which in many cases results in an observed zero return even though the actual one is non-zero. Similarly, financial prices are usually quoted with a few digits only (typically two), so financial returns are thus often measured as zero even though the true return is non-zero. This leads to the observation that an asset often exhibit more zeros when low-priced (in nominal terms), since a tick then corresponds to a higher return than when highly-priced. Accordingly, one may argue that zeros should be treated as missing values instead of zeros. Finally, impulse dummies are sometimes used to meancorrect returns in the conditional mean. This leads to mean-corrected returns equal to zero. When the impulse dummies are intended to neutralise the effect of large outliers or "jumps" - this is often the motivation in macroeconomics and finance, then one may argue

\footnotetext{
${ }^{4}$ However, Pantula (1986, p. 73) also stressed that it enables tests for integrated log-variance via Dickey and Fuller (1979) tests for unit roots.

${ }^{5}$ Another critique that has been directed towards the log-GARCH (e.g. Teräsvirta (2009)) is that the first unconditional autocorrelations of the squared returns, a measure of volatility persistence, can be unreasonably high. But this only occurs in very specific cases: The log-GARCH class allows for a much larger range of autocorrelation patterns than ordinary GARCH models, since the autocorrelation pattern depends on the shape of the conditional density (the more fat-tailed, the lower correlations) in addition to the persistence parameters.
} 
that the zeros should be treated as missing observations of actual returns. The second type of situation in which zero returns occur is when the zero-probability of actual return is truly non-zero. This is explored in Sucarrat and Grønneberg (2016). Here, therefore, our focus is exclusively on the first type of situation in which the zero probability is zero.

Estimators that do not rely on a specific distribution are greatly appreciated by practitioners, since then one needs not change the conditional density from application to application, or alternatively to use a sufficiently general and extra-parametrised density that makes estimation and inference more challenging numerically. Two types of such estimators have been proposed for the log-GARCH model, one "Standard" and one based on the ARMA representation. Francq et al. (2013) prove CAN under mild assumptions for a QMLE of the first type. In their estimator the density of conditional return is used for estimation, hence their estimator being Standard. For the second type, Sucarrat et al. (2016) exploit that the log-GARCH model admits an ARMA representation with Independent and Identically Distributed (IID) errors. Next, they show that CAN estimation of the ARMA representation by well-known methods provides CAN estimates of all the log-GARCH parameters, as long as the intercept bias in the log-volatility specification induced by the ARMA representation is appropriately adjusted for. Subsequently, another ARMA-based estimator was proposed by Francq and Sucarrat (2013). Their QMLE uses the centred exponential chi-squared distribution as instrumental density, which is more efficient when the conditional return is normal or close to normal. Both the Standard and the ARMA-based estimators are valid under mild assumptions, both types rely on the assumption that the probability of a zero return is zero and both types produce asymptotically biased estimates in the presence of zeros.

This paper makes three contributions. First, in Sections 2.2 and 2.3, we provide a framework for observed zero returns and propose an algorithm for unbiased estimation in their presence. The algorithm treats zeros as missing observations and replaces them with estimates of their conditional expectation. The algorithm is computationally simple and straightforwardly implemented with ARMA-based QMLEs and Least Squares Estimators (LSEs). ${ }^{6}$ However, it is not available for the Standard QMLE. Bondon and Bahamonde (2012) proposed an estimator for non-exponential ARCH models, i.e. an ARCH model without the important GARCH term, in the presence of missing observations. But to the best of our knowledge no such estimator has been put forward for GARCH models (neither exponential nor non-exponential). Second, we undertake an extensive set of Monte Carlo simulations (Section 3) to shed light on the effect of zeros, and to verify the conjectured asymptotic properties of our algorithm. If the algorithm we propose is not used when zeros are observed, then the simulations show that the downwards bias of volatility increases with the number of zeros, that the empirical standard errors are larger and that the reaction to volatility shocks is underestimated. The extent of these features depend on the parameter values, on the exact value used to replace zeros, on whether the conditional density is fat-tailed or not and on the type of estimator. By contrast, if the algorithm we propose is used, then the simulations show that the estimates are unbiased, and that the sample-size adjusted empirical standard error correspond well to their asymptotic counterparts. An additional simulation study suggests that we can

\footnotetext{
${ }^{6}$ The $R$ package lgarch implements the algorithm for several ARMA-based estimators, see Sucarrat (2014).
} 
usefully include estimated zero-probabilities as conditioning variables in a log-GARCH$\mathrm{X}$ model. Third, several empirical applications (Section 4) illustrate that the parameter estimates and the fitted conditional standard deviations can differ substantially in practice if zeros are not appropriately handled, and a case study of the Apple stock sheds further light on the relationship between return volatility, zeros and volume.

The rest of the paper is organised as follows. The next section, Section 2, provides an overview of the log-GARCH model and how estimation via the ARMA-representation is implemented. The section also contains the underlying framework that we rely upon and the details of our algorithm. Section 3 contains the Monte Carlo studies. Section 4 contains the empirical applications, whereas Section 5 concludes. Tables and Figures are located at the end.

\section{Model, framework and algorithm}

\subsection{The log-GARCH model}

If $\epsilon_{t}$ denotes financial return (possibly mean-corrected), then the $\log \operatorname{GARCH}(p, q)$ model is given by

$$
\begin{aligned}
\epsilon_{t} & =\sigma_{t} z_{t}, \quad z_{t} \sim \operatorname{IID}(0,1), \quad \operatorname{Pr}\left(z_{t}=0\right)=0, \quad \sigma_{t}>0, \\
\ln \sigma_{t}^{2} & =\alpha_{0}+\sum_{i=1}^{p} \alpha_{i} \ln \epsilon_{t-i}^{2}+\sum_{j=1}^{q} \beta_{j} \ln \sigma_{t-j}^{2}, \quad t \in \mathbb{Z},
\end{aligned}
$$

where $p$ is the ARCH order and $q$ is the GARCH order. Let $L$ denote the lag-operator and let $p^{*}=\max \{p, q\}$. A sufficient condition for the existence of a strictly stationary solution is that the roots of the lag polynomial $\left(1-\sum_{i=1}^{p^{*}}\left(\alpha_{i}+\beta_{i}\right) L^{i}\right)$ are all outside the unit circle, see Francq et al. (2013, example 2.2). For the $\log -\operatorname{GARCH}(1,1)$, this condition is $\left|\alpha_{1}+\beta_{1}\right|<1$. In the context of log-GARCH models, the socalled inlier issue (see Breidt and Carriquiry (1996) for a discussion in a Stochastic Volatility (SV) context) amounts to whether $E\left(\ln z_{t}^{2}\right)$ exists. For the Student's $t$ density and for the Generalised Error Distribution (GED), the two most common distributions in finance, $E\left(\ln z_{t}^{2}\right)$ generally exists. Francq et al. (2013) provide general conditions for the existence of log-moments.

It is well-known that (2) admits an ARMA representation, see e.g. Pantula (1986), Psaradakis and Tzavalis (1999) and Francq and Zakoïan (2006). Specifically, if $\left|E\left(\ln z_{t}^{2}\right)\right|<$ $\infty$, then adding $\ln z_{t}^{2}$ to each side of $(2)$, and then adding and subtracting $E\left(\ln z_{t}^{2}\right) \cdot(1-$ $\sum_{j=1}^{q} \beta_{j}$ ) to the right-hand side, yields (by re-arranging the terms) the $\operatorname{ARMA}(p, q)$ representation

$$
\ln \epsilon_{t}^{2}=\phi_{0}+\sum_{i=1}^{p} \phi_{i} \ln \epsilon_{t-i}^{2}+\sum_{j=1}^{q} \theta_{j} u_{t-j}+u_{t}, \quad u_{t}=\ln z_{t}^{2}-E\left(\ln z_{t}^{2}\right),
$$


where $q \leq p, u_{t} \sim I I D\left(0, \sigma_{u}^{2}\right)$,

$$
\phi_{0}=\alpha_{0}+\left(1-\sum_{j=1}^{q} \beta_{j}\right) \cdot E\left(\ln z_{t}^{2}\right), \quad \phi_{i}=\alpha_{i}+\beta_{i} \quad \text { and } \quad \theta_{j}=-\beta_{j} .
$$

Moreover, if $E\left[\left(\ln z_{t}^{2}\right)^{2}\right]<\infty$, then $\sigma_{u}^{2}<\infty$. It is well known that (3) admits a strictly stationary solution if the roots of the AR-polynomial $\mathcal{A}(L)=1-\phi_{1} L-\cdots-\phi_{p^{*}} L^{p^{*}}$ are all outside the unit cirle. In fact, this condition is exactly the same as the one above. In other words, consistent and asymptotically normal estimates of all the ARMA parameters - and hence all the log-GARCH parameters except the log-volatility intercept $\alpha_{0}$ - are thus readily obtained via usual ARMA estimation methods (e.g. the Gaussian QMLE or the LSE) subject to additional regularity conditions (most importantly, invertibility of the MA polynomial, and that the AR and MA polynomials have no common roots) ${ }^{7}$ see e.g. Brockwell and Davis (2006 [1991]). For a consistent estimate of $\alpha_{0}$, however, a consistent estimate of $E\left(\ln z_{t}^{2}\right)$ is needed. Sucarrat et al. (2016) derive

$$
-\ln \left[\frac{1}{T} \sum_{t=1}^{T} \exp \left(\widehat{u}_{t}\right)\right]
$$

as estimator of $E\left(\ln z_{t}^{2}\right)$, where the $\widehat{u}_{t}$ 's are the ARMA residuals, and provide conditions for its consistency and asymptotic normality. As a consequence, all the $\log \operatorname{GARCH}(p, q)$ parameters can be estimated consistently via the ARMA representation for a range of ARMA estimators, including the Gaussian QMLE and the LSE. Additional terms, e.g. asymmetry/leverage terms, or exogenous or predetermined conditioning information (i.e. "X"), can also be added without affecting the relationship between the log-GARCH and ARMA parameters, nor the structure of the bias-correction procedure. Francq and Sucarrat (2013) propose another ARMA-based QMLE that uses the centred exponential chi-squared distribution instead of the Gaussian as instrumental density. The motivation for this estimator is that it is asymptotically more efficient than the Gaussian ARMAQMLE when the conditional error $z_{t}$ is normal or close to normal. In the (empirical) presence of zeros, however, both are biased if zeros are replaced with a non-zero value $c$.

The Standard QMLE of Francq et al. (2013) undertakes estimation in terms of the conditional density of $\epsilon_{t}$, i.e. by maximising $\sum_{t=1}^{T} \ln f\left(\epsilon_{t} ; \sigma_{t}\right)$, where $f$ is the Gaussian density. Also, they use a slightly different version of the (symmetric) log-volatility specification. In their setup (2) is replaced by

$$
\ln \sigma_{t}^{2}=\alpha_{0}+\sum_{i=1}^{p} \alpha_{i} I_{\left\{z_{t-i} \neq 0\right\}} \ln \epsilon_{t-i}^{2}+\sum_{j=1}^{q} \beta_{j} \ln \sigma_{t-j}^{2}
$$

where $I_{\left\{z_{t-i} \neq 0\right\}}$ is an indicator function equal to 0 if $z_{t-i}=0$, and 1 otherwise. Of course, (2) and (6) are equal for all $t$ with probability 1 under the assumption that $\operatorname{Pr}\left(z_{t}=0\right)=0$. In empirical practice, however, (6) avoids the problem of possibly applying the natural

\footnotetext{
${ }^{7}$ For the $\log$-GARCH$(1,1)$, invertibility of the MA polynomial is equivalent to $\left|\beta_{1}\right|<1$, and the no common root assumption is equivalent to $\alpha_{1} \neq 0$.
} 
logarithm on zero values. Nevertheless, since the Standard QMLE also relies on the assumption $\operatorname{Pr}\left(z_{t}=0\right)=0$, the empirical presence of zeros also leads to biased estimates.

\subsection{Observed zeros - a framework}

If $\epsilon_{t}$ denotes the actual or true return, then the observed return $\tilde{\epsilon}_{t}$ is given by

$$
\tilde{\epsilon}_{t}=\epsilon_{t} I_{t}, \quad I_{t} \in\{0,1\} .
$$

Accordingly, the actual return $\epsilon_{t}$ is correctly observed whenever $I_{t}=1$, whereas it is incorrectly observed or "missing" whenever $I_{t}=0$. This approach is similar to that of Bondon and Bahamonde (2012). The process that determines true return $\epsilon_{t}$ we refer to as the Data Generating Process (DGP), whereas the process that determines $I_{t}$ we refer to as the Zero Generating Process (ZGP). If $I_{t}$ is non-random and known, then the main condition for the validity of our algorithm is usually satisfied. ${ }^{8}$ By contrast, if the ZGP is random, then the condition is not necessarily satisfied. Accordingly, our focus will henceforth be on the random case.

Let $\pi_{0 t}=\operatorname{Pr}_{t-1}\left(I_{t}=0\right)$ denote the (possibly) time-varying zero-probability conditional on the past. Of course, this means $\operatorname{Pr}_{t-1}\left(I_{t}=1\right)=\pi_{1 t}=1-\pi_{0 t}$. To fix ideas we will specify the zero probability as a dynamic logit model, but we are by no means restricted to this class of models. In the simplest case, therefore, when $I_{t}$ is IID, we have that $h_{t}=\rho_{0}$, where

$$
h_{t}=\ln \left(\pi_{1 t} / \pi_{0 t}\right), \quad \pi_{1 t}=\frac{1}{1+\exp \left(-h_{t}\right)} .
$$

For convenience we will sometimes refer to $\pi_{1 t}$ (and transformations thereof, e.g. $h_{t}=$ $\ln \left(\pi_{1 t} / \pi_{0 t}\right)$ ) as the zero-probability, since $\pi_{0 t}$ can straightforwardly be obtained via $\pi_{1 t}$ (and transformations thereof, e.g. $\pi_{0 t}=1-\pi_{1 t}$. Let $\mathcal{I}_{t-1}$ denote the set of past information, and let $\mathcal{I}_{t-1}^{z}=\left\{z_{t-1}, z_{t-2}, \ldots\right\}$ and $\mathcal{I}_{t-1}^{I}=\left\{I_{t-1}, I_{t-2}, \ldots\right\}$ with both $\mathcal{I}_{t-1}^{z} \subset \mathcal{I}_{t-1}$ and $\mathcal{I}_{t-1}^{I} \subset$ $\mathcal{I}_{t-1}$. Since the algorithm we propose replaces the missing values with the conditional expectations of the ARMA representation whenever $I_{t}=0$, the key condition for its validity is that

$$
E_{t-1}\left(\ln \epsilon_{t}^{2}\right)=E\left(\ln \epsilon_{t}^{2} \mid I_{t}, \mathcal{I}_{t-1}\right),
$$

where $E_{t-1}\left(\ln \epsilon_{t}^{2}\right)$ is the expectation of $\ln \epsilon_{t}^{2}$ conditional on the past associated with the ARMA representation. Little and Rubin (2002) distinguish between three cases, and we may provide a similar distinction adapted to the current setting:

1. Missing Completely at Random (MCAR): $z_{i}$ and $I_{j}$ are independent for all pairs $i, j$

2. Missing at Random (MAR): $z_{t}$ and $I_{t}$ are contemporaneously independent conditional on $\mathcal{I}_{t-1}$

3. Missing Not at Random (MNAR): $z_{t}$ and $I_{t}$ are contemporaneously dependent

\footnotetext{
${ }^{8} \mathrm{An}$ example where $I_{t}$ may be viewed as non-random and known is when zero daily returns are due to known closures of the financial trading venue in question.
} 
The algorithm we propose below will be valid for the MCAR and - by a straightforward extension - MAR cases, but not necessarily for the MNAR case.

In the MCAR case the ZGP is entirely independent of the DGP. Accordingly, condition (8) will hold if $\mathcal{I}_{t-1}=\mathcal{I}_{t-1}^{z} \cup \mathcal{I}_{t-1}^{I}$, and if the ARMA representation (3) is stationary and invertible, since then $E_{t-1}\left(\ln \epsilon_{t}^{2}\right)=E\left(\ln \epsilon_{t}^{2} \mid \mathcal{I}_{t-1}^{z}\right)=\psi_{0}+\sum_{i=1}^{\infty} \psi_{i} \ln z_{t-i}^{2}+E\left(\ln z_{t}^{2}\right)$. A straightforward example of MCAR is when $\epsilon_{t}$ is governed by (1)-(2) and when $I_{t}$ is IID. Another example is when $I_{t}$ is independent over time, but with a deterministic trend in the zero probability, e.g. $h_{t}=\rho_{0}+\rho_{1} \cdot t$. This model is of special interest, since it provides a simple description of a steady decrease (or increase) in the zero probability over time without an effect on return variability. We will return to variations of this model in Sections 3.3 and 4.2 .

In the MAR case the log-volatility $\ln \sigma_{t}^{2}$ can depend on past values of $I_{t}$. If this is the case, then condition (8) will not hold for the ARMA representation (3). However, the log-GARCH model - and hence the ARMA representation - can readily be extended to allow past values of $I_{t}$ to have an effect on $\ln \sigma_{t}^{2}$ by means of a log-GARCH-X specification. The log-GARCH-X model is given by

$$
\ln \sigma_{t}^{2}=\alpha_{0}+\sum_{i=1}^{p} \alpha_{i} \ln \epsilon_{t-i}^{2}+\sum_{j=1}^{q} \beta_{j} \ln \sigma_{t-j}^{2}+g\left(\lambda, x_{t-1}\right),
$$

where $g$ is a linear or nonlinear function of the exogenous or predetermined variables $x_{t-1}$, and a parameter vector $\lambda$. If $\operatorname{Pr}\left(z_{t}=0\right)=0$ and $\left|E\left(\ln z_{t}^{2}\right)\right|<\infty$, then (9) admits the ARMA-X representation

$$
\ln \epsilon_{t}^{2}=\phi_{0}+\sum_{i=1}^{p} \phi_{i} \ln \epsilon_{t-i}^{2}+\sum_{j=1}^{q} \theta_{j} u_{t-j}+g\left(\lambda, x_{t-1}\right)+u_{t}
$$

where the ARMA coefficients are related to the log-GARCH coefficients in the same ways as before, i.e. by (4), and where $u_{t}$ is the same as earlier, i.e. $u_{t}=\ln z_{t}^{2}-$ $E\left(\ln z_{t}^{2}\right)$. Accordingly, with suitable assumptions on the $x_{t}$, the log-GARCH-X model can be estimated via the ARMA-X representation, see Sucarrat et al. (2016). Examples of X-variables of interest include leverage, volatility proxies, volume and past values of $I_{t}$ (or transformations thereof, e.g. past values of $h_{t}$ ). The log-GARCH-X specification is therefore particularly interesting in the current context, since it provides a framework in which the effects of past observed zeros, volume, etc. on volatility can be studied jointly. Let the set of past information now be given by $\mathcal{I}_{t-1}=\mathcal{I}_{t-1}^{z} \cup \mathcal{I}_{t-1}^{X}$, where $\mathcal{I}_{t-1}^{z}=\left\{z_{t-1}, z_{t-2}, \ldots\right\}, \mathcal{I}_{t-1}^{X}=\left\{x_{t-1}, x_{t-2}, \ldots\right\}$ and $\mathcal{I}_{t-1}^{I} \subset \mathcal{I}_{t-1}^{X}$. In other words, the conditioning $\mathrm{X}$-vector contains the past information associated with the zero-process. If $E_{t-1}\left(\ln \epsilon_{t}^{2}\right)=E\left(\ln \epsilon_{t}^{2} \mid \mathcal{I}_{t-1}\right)=\phi_{0}+\sum_{i=1}^{p} \phi_{i} \ln \epsilon_{t-i}^{2}+\sum_{j=1}^{q} \theta_{j} u_{t-j}+g\left(\lambda, x_{t-1}\right)$ is equal to $E_{t-1}\left(\ln \epsilon_{t}^{2} \mid I_{t}, \mathcal{I}_{t-1}\right)$, then condition (8) holds.

In the MNAR case condition (8) may not hold, since $z_{t}$ and $I_{t}$ are contemporaneously dependent. This implies that also $\epsilon_{t}$ and $I_{t}$ are contemporaneously dependent. If observed zeros are due to discrete prices or rounding errors, then this seems to suggest that missingness depends on the true return being close to zero, i.e. that $\epsilon_{t}$ and $I_{t}$ are contemporaneously dependent. However, this is not necessarily the case. Define the true 
$\log$-return as $\epsilon_{t}=\left(\ln P_{t}-\ln P_{t-1}\right)$, so that the true price at $t$ is given by $P_{t}=P_{t-1} \cdot e^{\epsilon_{t}}$. A zero return is thus observed whenever the rounded price $\widetilde{P}_{t}$ is equal to the rounded price $\widetilde{P}_{t-1}$. Clearly the occurrence of zeros depends on a range of factors, including the degree of discreteness (e.g. the tick-size), the nominal level of the price (i.e. the lower price, the more likely a zero will be observed) ${ }^{9}$ the level of conditional volatility (i.e. the higher, the less likely a zero will be observed), the dynamics of volatility (e.g. the sensitivity to shocks) and the value of $\left|z_{t}\right|$. Accordingly, zeros due to rounding are not necessarily due to small values of $\left|z_{t}\right|$. However, if they are, then condition (8) may not hold, since this then implies a contemporaneous dependence between $z_{t}$ and $I_{t}$.

\subsection{Algorithm}

The actual return $\epsilon_{t}$ is correctly observed whenever $I_{t}=1$ in (7). Whenever $I_{t}=0$, then the actual return $\epsilon_{t}$ is missing. A common approach to missing observations is the Expectation-Maximisation (EM) algorithm popularised by Dempster et al. (1977). There, missing values are handled in what they characterised as two separate steps: The Expectation or E-step and the Maximisation or M-step. A common approach to missing observations in an ARMA context is state-space models, see e.g. Jones (1980), Shumway and Stoffer (1982), Kohn and Ansley (1986), and Gomez and Maravall (1994). Shumway and Stoffer (1982) combines the state-space approach with the EM-algorithm. A third approach to missing observations exploits the specific structure of an ARMA model without putting it into state-space form. An example of this approach is Wincek and Reinsel (1986).

The algorithm we propose is in the spirit of the EM-algorithm, since it can be viewed as maximising the approximate conditional (quasi) log-likelihood while replacing the missing values by estimates of their conditional expectations. However, the separation between the $\mathrm{E}$ and $\mathrm{M}$ steps is not as sharp as usual, and it exploits the recursive nature of the ARMA model. The algorithm holds several advantages compared to those cited above. First, it is conceptually simpler than state-space approaches, since we do not cast the ARMA model into its state-space form. Second, our algorithm is computationally simpler, since we work with the approximate conditional log-likelihood. Third, the key condition (8) holds for a much larger range of ZGPs. In particular, it holds for certain MAR schemes, where conditional volatility depends on past zeros. Fourth, it is valid for several ARMA-based estimators. For concreteness we outline our algorithm for the Gaussian QMLE only, but it can straightforwardly be adapted to the LSE and the centred exponential Chi-squared QMLE of Francq and Sucarrat (2013) as well. The next section, Section 3, contains simulations that supports that the conjectured asymptotic properties of the algorithm in combination with the Gaussian QMLE are retained in the presence of zeros.

Let $\widehat{\phi}_{0}^{(k)}, \widehat{\phi}_{1}^{(k)}, \ldots, \widehat{\phi}_{p}^{(k)}$ and $\widehat{\theta}_{1}^{(k)}, \ldots, \widehat{\theta}_{q}^{(k)}$ denote the parameter estimates of the ARMA representation (3) after $k$ iterations with some numerical method (e.g. Newton-Raphson). The initial parameter estimates are given at $k=0$. If there are no observed zeros, then at the $k$ th. iteration the numerical method thus proceeds in the usual way:

\footnotetext{
${ }^{9}$ For example, suppose the true return at $t$ is $+0.25 \%$. If prices are rounded to two decimals, then $P_{t-1}=1$ will result in an observed zero return at $t$, that is, $\ln \widetilde{P}_{t}-\ln \widetilde{P}_{t-1}$ will be zero. By contrast, if $P_{t-1}=10$, then the observed return at $t$ will be zon-zero.
} 
1. Compute, recursively, for $t=1, \ldots, T$ :

$$
\widehat{u}_{t}^{(k-1)}=\ln \epsilon_{t}^{2}-\widehat{\phi}_{0}^{(k-1)}-\sum_{i=1}^{p} \widehat{\phi}_{i}^{(k-1)} \ln \epsilon_{t-i}^{2}-\sum_{j=1}^{q} \widehat{\theta}_{j}^{(k-1)} \widehat{u}_{t-j}^{(k-1)},
$$

where $\widehat{u}_{t}^{(k-1)}$ is an estimate of $u_{t}$, and where the initial values $\ln \epsilon_{0}^{2}, \ldots, \ln \epsilon_{-p+1}^{2}$ and $u_{0}, \ldots, u_{-q+1}$ are replaced by estimates at each iteration $k$.

2. Compute the approximate conditional log-likelihood $\sum_{t=1}^{T} \ln f\left(\widehat{u}_{t}^{(k-1)} ; \widehat{\sigma}_{u}^{(k-1)}\right)$, where $f$ is the univariate Gaussian density and $\widehat{\sigma}^{(k-1)}$ the estimate of its scale parameter, and other quantities (e.g. the gradient and/or Hessian) needed by the numerical method to generate $\widehat{\phi}_{0}^{(k)}, \widehat{\phi}_{1}^{(k)}, \ldots, \widehat{\phi}_{p}^{(k)}, \widehat{\theta}_{1}^{(k)}, \ldots, \widehat{\theta}_{q}^{(k)}$ and $\widehat{\sigma}^{(k)}$.

The algorithm we propose modifies this procedure in several ways. Let $\tau$ denote the locations of the non-zero values of $\epsilon_{t}$, and let $T^{*}$ denote the number of non-zero values. The $k$ th. iteration now proceeds as follows:

1. Compute, recursively, for $t=1, \ldots, T$ :

a) ${\overline{\ln \epsilon_{t}^{2}}}^{(k-1)}=\left\{\begin{array}{l}\ln \epsilon_{t}^{2} \quad \text { if } t \in \tau \\ \widehat{E}_{t-1}\left(\ln \epsilon_{t}^{2}\right)^{(k-1)} \quad \text { if } t \notin \tau\end{array}\right.$

where

$$
\begin{aligned}
& \widehat{E}_{t-1}\left(\ln \epsilon_{t}^{2}\right)^{(k-1)}=\widehat{\phi}_{0}^{(k-1)}+\sum_{i=1}^{p} \widehat{\phi}_{i}^{(k-1)}{\overline{\ln \epsilon^{2}}}_{t-i}^{(k-1)}+\sum_{j=1}^{q} \widehat{\theta}_{j}^{(k-1)} \widehat{u}_{t-j}^{(k-1)} \\
& \text { b) } \widehat{u}_{t}^{(k-1)}={\overline{\ln \epsilon_{t}^{2}}}^{(k-1)}-\widehat{\phi}_{0}^{(k-1)}-\sum_{i=1}^{p} \widehat{\phi}_{i}^{(k-1)} \frac{\ln ^{2}}{t-i}(k-1)-\sum_{j=1}^{q} \widehat{\theta}_{j}^{(k-1)} \widehat{u}_{t-j}^{(k-1)} \text {. }
\end{aligned}
$$

The initial values $\ln \epsilon_{0}^{2}, \ldots, \ln \epsilon_{-p+1}^{2}$ and $u_{0}, \ldots, u_{-q+1}$ are replaced by estimates at each iteration $k$.

2. Compute the approximate conditional log-likelihood $\sum_{t \in \tau} \ln f\left(\widehat{u}_{t}^{(k-1)} ; \widehat{\sigma}_{u}^{(k-1)}\right)$, where $f$ is the univariate Gaussian density and $\widehat{\sigma}_{u}^{(k-1)}$ the estimate of its scale parameter, and other quantities (e.g. the gradient and/or Hessian) needed by the numerical method to generate $\widehat{\phi}_{0}^{(k)}, \widehat{\phi}_{1}^{(k)}, \ldots, \widehat{\phi}_{p}^{(k)}, \widehat{\theta}_{1}^{(k)}, \ldots, \widehat{\theta}_{q}^{(k)}$ and $\widehat{\sigma}^{(k)}$.

Step 1.a) means the value of $\ln \epsilon_{t}^{2}$ is replaced by an estimate of the conditional expectation $E_{t-1}\left(\ln \epsilon_{t}^{2}\right)$ at zero locations. This estimate does not rely on any specific assumption on the density of $\ln z_{t}^{2}$ (say, Gaussianity), nor on the density of $z_{t}$, apart from $\operatorname{Pr}\left(z_{t}=0\right)=0$ and the existence of $E\left(\ln z_{t}^{2}\right)$. However, it is worth noting that, at the population level, the value $E_{t-1}\left(\ln \epsilon_{t}^{2}\right)$ is not only the value that minimises the conditional forecast error variance, it is also the value that maximises the conditional expected log-likelihood $E_{t-1}\left[\ln f\left(u_{t} ; \sigma_{u}\right)\right]$ at $t$ if $f$ is the Gaussian density. In other words, if the Gaussian distribution is used as the instrumental density in a QMLE, then there is a clear link to the EM-algorithm, where missing values are estimated by maximising the expected loglikelihood conditional on the observed data, see Dempster et al. (1977, page 6). In Step 
1.b) the recursion value $\widehat{u}_{t}^{(k-1)}$ is, by construction, equal to 0 at the zero-locations. This has implications for Step 2, where the symbolism $t \in \tau$ means the log-likelihood only includes contributions from non-zero locations. An important practical implication of this is that likelihood comparisons with competing models should be in terms of the average log-likelihood with division by $T^{*}$ rather than $T$. After estimation of the ARMA representation the ARMA residuals $\widehat{u}_{t}$ at non-zero locations are used to estimate $E\left(\ln z_{t}^{2}\right)$ with (5). Next, estimates of the log-GARCH parameters are obtained via the formulas in (4). The algorithm we have outlined is valid for the MCAR case. However, by straightforward modifications, i.e. replacing the log-GARCH and ARMA expressions with log-GARCH-X and ARMA-X expressions, respectively, the algorithm can also be applied to log-GARCH$\mathrm{X}$ models when zeros occur according to the MAR scheme. In the Standard QMLE the algorithm is not applicable. The reason for this is that an estimate of $\ln \epsilon_{t}^{2}$ is needed as a replacement for the missing observations in the recursion of the log-volatility specification (6), and this is not provided by the estimator when it is interpreted as a QMLE. If the Standard QMLE is interpreted as an exact MLE, however, then a similar algorithm to the one above can be used. In that case $z_{t}$ is standard normal and $E\left(\ln z_{t}^{2}\right)=-1.27$.

It is well known that both the Gaussian QMLE and the LSE produce consistent and asymptotically normal estimates of the ARMA parameters under mild assumptions when there are no missing values, see e.g. Hannan (1973), and Brockwell and Davis (2006 [1991]) (Francq et al. (2011), and the references therein, contain more recent and general results). In particular, it is not required that the initial values in the recursion (11) are equal to their true values $\ln \epsilon_{0}^{2}, \ldots, \ln \epsilon_{-p+1}^{2}, u_{0}, \ldots, u_{-q+1}$. This can be referred to as an "irrelevance of initial values" condition. If $\widetilde{u}_{t}$ denotes the ARMA error at the true parameter values but having started at $t=1$ (i.e. not in the infinite past) with some arbitrary initial values on $\ln \epsilon_{t}^{2}$ and $u_{t}$, then the irrelevance of initial values condition means the difference between $u_{t}$ and $\widetilde{u}_{t}$ becomes sufficiently small in some appropriate sense as $t \rightarrow \infty$. Heuristically, our algorithm can be viewed as repeatedly - instead of

only once - creating an initial value issue, since the true value of $\ln \epsilon_{t}^{2}$ is replaced by its conditional expectation whenever $I_{t}=0$. In other words, whenever $I_{t}=0$, then the $\widetilde{u}_{t}$ is perturbed away from $u_{t}$. This suggests zeros cannot occur too often, since - heuristically $\widetilde{u}_{t}$ may need sufficient time to converge back towards $u_{t}$ before it is perturbed away again. Otherwise the cumulated difference may not be asymptotically irrelevant. How large the zero-probability can be before the cumulated discrepancy becomes relevant (in some appropriate sense), however, is not clear. The perturbation is minimal in the conditional variance sense, since the missing values are replaced by their conditional expectations. So a reasonable conjecture is that the probability can be sufficiently large to be of practical interest (in daily financial data the zero-proportion is usually between 0 and 0.05 ). This is certainly supported by the simulations in Section 3.

\section{A Monte Carlo study}

\subsection{Effect of zeros}

To shed light on the effect of observed zeros on parameter estimates we compare the Standard QMLE and the Gaussian ARMA-QMLE in a simulation experiment. In the 
experiment the DGP of return $\epsilon_{t}$ is given by the $\log \operatorname{GARCH}(1,1)$ specification

$$
\epsilon_{t}=\sigma_{t} z_{t}, \quad z_{t} \sim \operatorname{IID}(0,1), \quad \ln \sigma_{t}^{2}=\alpha_{0}+\alpha_{1} \ln \epsilon_{t-1}^{2}+\beta_{1} \ln \sigma_{t-1}^{2},
$$

for empirically relevant combinations of the parameters $\alpha_{0}, \alpha_{1}$ and $\beta_{1}$. These combinations are referred to as $\mathrm{A}, \mathrm{B}$ and $\mathrm{C}$. The zero probability is constant over time and equal to either $0,0.05,0.10$ or 0.20 . In other words, the ZGP is random and entirely independent of the DGP, i.e. the MCAR case (we relax this assumption in Section 3.3).

For the Gaussian ARMA-QMLE estimation is undertaken with the adjusted return

$$
\tilde{\tilde{\epsilon}}_{t}=\left\{\begin{array}{l}
\epsilon_{t} \text { if } I_{t}=1, \\
c \text { if } I_{t}=0, \quad c>0 .
\end{array}\right.
$$

The $\log$-volatility specification is thus $\ln \sigma_{t}^{2}=\alpha_{0}+\alpha_{1} \ln \tilde{\tilde{\epsilon}}_{t-1}^{2}+\beta_{1} \ln \sigma_{t-1}^{2}$. Clearly the choice of $c$ will influence the results. In particular, among the natural choices of $c$, i.e. values between the numerical minimum of the statistical software in question and, say, 0.1 , the closer to zero, the larger the bias is likely to be in our experience. Moreover, the closer to 0 , the more often numerical issues (e.g. non-convergence) are encountered. As an intermediate choice we therefore choose $c=0.01$ for the simulations. For the Standard QMLE the zeros of observed return $\tilde{\epsilon}_{t}$ are simply not included in the recursion because of the indicator function in the log-volatility specification (6). It is worth noting, however, that this is equivalent of setting $c=1$ in (16). In other words, any difference in simulation result is not only due to the estimator, but also due to the different value of $c$.

Table 1 contains the results, and Figures 1-2 provides a comparison with the algorithm (see Section 3.2) for parameter combinations A and C. We do not report the graphs for $\mathrm{B}$, since they are very similar to those of C. For the Standard QMLE the effect of zeros is straightforward: The higher the zero probability, the greater the bias, and the bias is almost invariably equal or higher when the conditional density is fat-tailed (i.e. standardised $t(5))$. The log-volatility intercept $\alpha_{0}$ is biased downwards, which means volatility will generally be biased downwards in the presence of zeros. The ARCH parameter $\alpha_{1}$, which controls the impact of shocks on volatility, is also biased downwards. The presence of zeros thus means volatility will be under-responsive to shocks. This effect is exacerbated by the upward bias of $\beta_{1}$, since this parameter controls the effect of the long-term component of volatility. Finally, increasing the zero probability increases the standard errors.

For the Gaussian ARMA-QMLE the biases are generally bigger compared with the Standard QMLE, but not always as straightforward. This is most readily seen in the Figures. Higher zero probability means larger negative bias for both $\alpha_{0}$ and $\alpha_{1}$, although the bias is not always larger for $\alpha_{0}$ when compared with those of the Standard QMLE. For $\beta_{1}$ the effect of zeros is more complex since the bias can change sign. Finally, also for the Gaussian ARMA-QMLE do the empirical standard increase when the zero probability increases, but the increase can be much bigger.

\subsection{Properties of algorithm}

To study the properties of the Gaussian ARMA-QMLE in combination with our algorithm we conduct two experiments. The first is similar to the one in the previous subsection 
in that the ZGP is IID, whereas in the second the zero probability decreases over time. Both experiments corresponds to the MCAR case, where the ZGP is entirely independent of the DGP.

The results of the first experiment are contained in Panel 1 of Table 2, and Figures 1-2 compare the finite sample bias with those of Section 3.1. It is clear that the algorithm corrects the bias for all three parameters. The finite sample biases increase slightly (and more so for $\left.z_{t} \sim t(5)\right)$ as the zero probability increases, but this is not surprising since more observations are lost by treating zeros as missing values when the zero probability increases. The empirical standard errors are virtually unaffected as the the zero probability increases, which is in stark contrast to the QMLEs without the algorithm. Moreover, the empirical standard error correspond well to their (adjusted) asymptotic counterparts. Finally, compared with the Standard QMLE the finite sample bias is substantially smaller for the location-parameter $\alpha_{0}$, i.e. the most important parameter in determining the level of volatility.

In the second experiment we study the properties of our algorithm in combination with the Gaussian QMLE when the zero probability is steadily decreasing. This is in line with the empirical observation that the zero probability falls over time, e.g. due to increased liquidity and/or volume, reduced discreteness/smaller ticks, higher nominal prices and other changes in how markets operate. As earlier we specify the DGP as a $\log$-GARCH $(1,1)$ with the same parameter values as earlier, i.e. A, B and C. The ZGP is entirely independent of the DGP, with the zero probability steadily falling according to a dynamic logit model. Let "relative" time be given by $t / T$ for $t=0,1,2, \ldots, T$ such that $t / T \in[0,1]$. The ZDP is then given by the dynamic logit-model

$$
h_{t}=\rho_{0}+\rho_{1} \cdot(t / T),
$$

where $h_{t}=\ln \left(\pi_{1 t} / \pi_{0 t}\right), \pi_{1 t}=1 /\left(1+\exp \left(-h_{t}\right)\right)$ and $\rho_{0}=1.9, \rho_{1}=3.4$. The values of $\rho_{0}$ and $\rho_{1}$ are chosen on the basis of the empirical estimates in Section 4.2. This means the zero-probability is given by $\pi_{0 t}=1-\pi_{1 t}=0.130$ at the beginning of the sample (i.e. $t=0)$, and by $\pi_{0 t}=0.005$ at the end (i.e. $\left.t=T\right)$.

The results are contained in Panel 2 of Table 2. On average, the zero proportion $\widehat{\pi}_{0}$ produced by the ZGP given by (17) is about 0.04, and the properties of the Gaussian ARMA-QMLE w/algorithm is therefore virtually identical to the results in Panel 1 when $\pi_{0}=0.05$. The biases are corrected, and the empirical standard errors correspond well to their (adjusted) asymptotic counterparts.

\subsection{Can we condition on estimated zero-probabilities?}

An X-variable of special interest is the zero-probability itself (or transformations thereof). Of course, in empirical practice one is unlikely to have access to the true zero-probabilities. To shed light on whether one in practice may (usefully) try to model volatility in terms of estimated past zero-probabilities, we device an experiment where the true return depends on (transformations of) past zero probabilities (i.e. we are in the MAR case). Specifically, 
the ZDP is given by (17), but the DGP is given by the log-GARCH(1,1)-X specification

$$
\ln \sigma_{t}^{2}=\alpha_{0}+\alpha_{1} \ln \epsilon_{t-1}^{2}+\beta_{1} \ln \sigma_{t-1}^{2}+\lambda h_{t-1}
$$

where $\lambda=-0.1$, and where the combinations of $\alpha_{0}, \alpha_{1}$ and $\beta_{1}$ are the same as in the previous simulations (i.e. $\mathrm{A}, \mathrm{B}$ and $\mathrm{C}$ ). The results, which are available in the supplementary material, suggests estimates of $h_{t}$ as conditioning variables are capable of proxying their true values reasonably well, even for small (in financial contexts) samples.

\section{Empirical applications}

This section contains two empirical applications. In the first our objective is simply to illustrate how much volatility estimates can differ if zero-returns are not properly handled. In the second we illustrate how a log-GARCH-X model can be exploited to shed further light on the relationship between return volatility, observed zeros and volume.

\subsection{The effect of zero-returns on volatility}

We compare the difference in parameter estimates and fitted conditional standard deviations for six daily financial returns: The Apple and Ekornes stocks (more information on Ekornes shortly), the Standard and Poor's 500 stock market index (SP500), the EUR/USD exchange rate, the WTI oil price and the London gold price. These account for a variety of market characteristics. For example, whereas the EUR/USD is traded in a global market almost continuously 24-hours a day and seven days a week - possibly with thousands of trades per second, the London Gold price is only fixed twice a day, and presumably not on Bank holidays and in weekends. In that regard it should be noted that we exclude weekends from our analysis for all six returns (i.e. zeros are not due to weekends). Our interest in the Ekornes stock price return is due to its relatively large proportion of zeros (about 19\%), and the main reason for the zeros is non-trading (i.e. a zero volume). Ekornes is a leading Nordic furniture manufacturer that is listed on the Oslo Stock Exchange. It can be described as a medium-sized company in international terms, since its market value is approximately 300 million euros at the end of the sample. The sources of the data are Yahoo Finance (http://finance.yahoo.com/) for the Apple, Ekornes and SP500 series, the European Central Bank (http://www.ecb.int/) for the EUR/USD series, the US Energy Information Agency (http://www.eia.gov/) for the WTI crude oil price (in USD) per barrel series and Kitco (http://www.kitco.com/) for the London afternoon (i.e. PM) gold price series.

The sample dates and descriptive statistics of the returns are contained in the upper part of Table 3. The returns exhibit the usual properties of excess kurtosis compared with the normal, and ARCH as measured by the first order autocorrelation in the squared return. The number of zeros varies from only 2 observations (about $0.1 \%$ of the sample) for SP500 to 667 observations (about 19\% of the sample) for Ekornes. The reasons for each zero are likely to differ substantially both within and across markets. We do not try to identify these reasons, since our main objective is to illustrate how the estimates and fitted conditional standard deviations differ according to estimation method. 
The middle part of Table 3 contains the estimates of the $\log$ - GARCH(1,1) specification

$$
\epsilon_{t}=\sigma_{t} z_{t}, \quad \ln \sigma_{t}^{2}=\alpha_{0}+\alpha_{1} \ln \epsilon_{t-1}^{2}+\beta_{1} \ln \sigma_{t-1}^{2},
$$

where $\epsilon_{t}$ is the log-return in percent (i.e., the log-difference of the financial price multiplied by 100). We use two estimators. In the first, labelled 0 -adj, we replace zero returns by the minimum of the absolute non-zero value, and then estimate with the Gaussian ARMAQMLE. In the second, labelled Algo, zeros are kept and then the algorithm proposed in Section 2.3 is used in combination with the Gaussian ARMA-QMLE. Unsurprisingly, the biggest numerical differences in the parameter estimates are produced by Ekornes (highest number of zeros; $19 \%$ of the sample), and the smallest are produced by SP 500 (only two zeros; $0.1 \%$ of the sample). The estimates of the intercept $\alpha_{0}$, which controls the unconditional variance, are always higher for the algorithm, apart from Ekornes. This is somewhat surprising, due to the high number of zeros for Ekornes. There clearly seems to be some interaction with the persistence parameter $\beta_{1}$, since it is unusually low, 0.784 , when zeros are replaced with the minimum of non-zero returns. Similarly, the algorithm estimate of $\beta_{1}$ for Ekornes is the only one that is substantially higher (0.943). In the other five cases the algorithm estimates are slightly lower. With respect to the estimate of the ARCH parameter $\alpha_{1}$, which controls the short-term impact of shocks or large (in absolute value) returns at $t-1$, the estimates of the algorithm are always higher - in most cases substantially, except for Ekornes (in which it is lower). Finally, the residual diagnostic test, a Ljung and Box (1979) test of second-order autocorrelation in the squared standardised residuals $\left(\widehat{z}_{t}^{2}\right)$, suggests the algorithm provides an improvement in all six cases, in the sense that the test-statistic falls. However, the significance results do not suggest that a plain $\log$ - $\mathrm{GARCH}(1,1)$ specification is fully adequate. In all but one case (Gold) is the null of no second-order autocorrelation rejected at the usual significance levels. We explore possible reasons in relation with the estimates of the asymmetric models (below).

Descriptive statistics of the fitted conditional standard deviations are contained in Table 4, and graphs of their ratios are contained in Figure 3. They clearly suggest that estimation method can matter a lot, both nominally and in relative terms. For example, for Apple the algorithm yields fitted conditional standard deviations that are up to 2.15 times higher, and the maximum nominal difference is 1.9. Such differences can make a huge difference in risk analysis and asset pricing. The Apple graphs also reveals what seems to be an inverse tendency. In the beginning of the sample the algorithm produces higher fitted conditional standard deviations. However, this is reversed in the second part of the sample. A possible reason is that there are fewer zeros in the second part of the sample (see the graph of $I_{t}$ in Figure 4). For most returns the average fitted conditional standard deviation is higher for the algorithm. The only case where the average difference is not positive is oil. There, the average is negative, but very close to zero $(-0.008)$. The ratio graph, however, clearly shows that, in relative terms, the algorithm occasionally produce values that are up to $66 \%$ higher. So, all in all, the comparison of fitted conditional standard deviations show that the algorithm generally produces higher values, and sometimes much higher.

Financial returns often exhibit asymmetry (i.e. leverage). To explore the effect of zeros on asymmetry we also estimate a $\log -\mathrm{GARCH}(1,1)$ specification with an asymmetry 
term equivalent to that of Francq et al. (2013). Specifically, for each of the six returns we estimate

$$
\epsilon_{t}=\sigma_{t} z_{t}, \quad \ln \sigma_{t}^{2}=\alpha_{0}+\alpha_{1} \ln \epsilon_{t-1}^{2}+\beta_{1} \ln \sigma_{t-1}^{2}+\lambda_{1}\left(\ln \epsilon_{t-1}^{2}\right) I_{\left\{\epsilon_{t-1}<0\right\}},
$$

where $I_{\left\{\epsilon_{t}<0\right\}}$ is and indicator function equal to 1 at $t$ if $\epsilon_{t}<0$, and 0 otherwise. ${ }^{10}$ We use the same estimation procedures as in the symmetric case above. The estimation results are contained in bottom part of Table 3, and they show that the differences between the 0 -adj and Algo estimates are almost identical to the symmetric case: In all but one case (Ekornes) is the intercept estimate $\widehat{\alpha}_{0}$ equal to or higher for Algo, in all but one case (Ekornes) is the ARCH estimate $\widehat{\alpha}_{1}$ higher for Algo, and in all but one case (Ekornes) is the GARCH estimate $\widehat{\beta}_{1}$ lower for Algo. As to whether there is asymmetry or not, approximate tests can be conducted by using the approximate standard errors. In the Algo case, the $t$-ratios (in absolute value) for Apple, Ekornes and Oil are 3.83, 4.67 and 2.71, respectively. This suggests these returns exhibit asymmetry. For the other three return series the $t$-ratios (in absolute value) are 1.67 or lower. In the 0 -adj case, only Ekornes and Oil exhibit asymmetry ( $t$-ratios of 2.52 and 3.00, respectively), as the $t$-ratios of the others are 1.67 or lower in absolute value. The parameter estimates for EUR/USD can be compared with those of Francq et al. (2013). Re-parametrised for comparison, the latter's estimates are: $\widehat{\alpha}_{0}=0.024, \widehat{\alpha}_{1}=0.027, \widehat{\beta}_{1}=0.971$ and $\widehat{\lambda}_{1}=-0.011$. That is, closer to the estimates of Algo than those of 0 -adj in nominal terms. Finally, just as in the symmetric case, the test statistics for second order $\mathrm{ARCH}$ in the standardised residuals $\widehat{z}_{t}$ are better (i.e. lower) for Algo. However, even though the asymmetry term appears to improve the diagnostics in several cases, the null of no $\mathrm{ARCH}$ is still rejected at usual significance levels for Apple, Ekornes, SP500 and Oil (EUR/USD is at the border with a $p$-value of $10 \%)$. This may be due to structural breaks, or the fact that we have not mean-corrected returns (zeros disappear if we mean-correct), or that a higher order log-GARCH specification is needed. Another possibility is that the diagnostic test we use has not been adequately modified to handle missing values (observations containing zeros are removed).

A comparison of the fitted conditional standard deviations reveals that they are very similar to the symmetric case. Accordingly, we do not report these, but they are available in the supplementary material.

\subsection{Apple: Return volatility, zeros and volume}

The proportion of observed zeros changes over time. Including past zero probabilities (and/or transformations thereof) and other variables to the X-vector in a log-GARCH-X model enables us therefore to study the impact on volatility in more detail. Here, we

\footnotetext{
${ }^{10}$ The stability condition is more complicated in the asymmetric case, see Francq et al. (2013). Specifically, in our parametrisation, under the assumption that $\alpha_{1}, \beta_{1}>0$, the condition is $\mid \alpha_{1}+\lambda_{1} I_{\left\{\lambda_{1}<0\right\}}+$ $\left.\beta_{1}\right|^{\operatorname{Pr}\left(z_{t}>0\right)} \cdot\left|\alpha_{1}-\lambda_{1} I_{\left\{\lambda_{1}>0\right\}}+\beta_{1}\right|^{1-\operatorname{Pr}\left(z_{t}>0\right)}<1$, where $\lambda_{1}=\left(\alpha_{1+}-\alpha_{1-}\right), I_{\left\{\lambda_{1}<0\right\}}$ is an indicator variable equal to 1 if $\lambda_{1}<0$ and 0 otherwise, and $I_{\left\{\lambda_{1}>0\right\}}$ is an indicator variable equal to 1 if $\lambda_{1}>0$ and 0 otherwise. All the models in the bottom panel of Table 3 satisfy this condition under the assumption that the density of $z_{t}$ is symmetric (i.e. $\operatorname{Pr}\left(z_{t}>0\right)=1 / 2$ ).
} 
illustrate this by a simple case study of the Apple stock return.

Figure 4 contains graphs of the Apple stock price, return, $I_{t}$ (i.e. $I_{t}=0$ means return is zero on day $t$ ), volume (in USD) and log-volume. The graph of $I_{t}$ clearly shows that the occurrence of zeros is less likely towards the end of the sample. Similarly, the volume graph reveals that volume is higher in the second half of the sample, and the price graph reveals that the nominal price is increasing over the sample. To shed light on whether the increase in volume or nominal price is indeed one of the reasons for the fall in zero-probability, we estimate the four dynamic logit models

$$
\begin{aligned}
h_{t} & =\rho_{0}, \\
h_{t} & =\rho_{0}+\rho_{1} \cdot(t / T), \\
h_{t} & =\rho_{0}+\rho_{1} \ln V_{t}, \\
h_{t} & =\rho_{0}+\rho_{1} \ln P_{t},
\end{aligned}
$$

where $h_{t}=\ln \left(\pi_{1 t} / \pi_{0 t}\right)$ and $\pi_{1 t}=1 /\left(1+\exp \left(-h_{t}\right)\right)$. In the first the zero-probability is constant, in the second the zero-probability is determined by a time-trend, in the third volume determines the zero-probability, whereas in the fourth the nominal price $P_{t}$ determines the zero probability. To recall, the last model is motivated by the fact that higher (nominal) prices often results in fewer zeros than when an asset is low-priced for the same tick-size. Table 5 contains the estimation results. The latter three models produce a substantially higher log-likelihood than the first, and among the latter three the log-likelihood of the volume-model is slightly higher. This can be interpreted to suggest that increased volume rather than discreteness (i.e. fewer zero due to higher nominal prices) is the main reason for the downwards trend in the zero probability.

To shed light on whether zeros and volume have an effect on volatility, we estimate four volatility models that are all contained in

$$
\ln \sigma_{t}^{2}=\alpha_{0}+\alpha_{1} \ln \epsilon_{t-1}^{2}+\beta_{1} \ln \sigma_{t-1}^{2}+\lambda_{1}\left(\ln \epsilon_{t-1}^{2}\right) I_{\left\{\epsilon_{t-1}<0\right\}}+\lambda_{2} \ln V_{t-1}+\lambda_{3} I_{t-1}+\lambda_{4} \widehat{h}_{t-1},
$$

where $\widehat{h}_{t}$ are the fitted values of (23). We use the Gaussian ARMA-QMLE in combination with the algorithm proposed in Section 2.3, and Table 6 contains the estimation results. Unsurprisingly, the constant volatility model has a much smaller log-likelihood than the three other models, and fares worse according to the Schwarz (1978) Information Criterion (BIC). Maybe somewhat surprisingly, however, is that the lags of $\ln V_{t}, I_{t}$ and $\widehat{h}_{t}$ do not improve the fit in terms of BIC compared with the asymmetric log-GARCH $(1,1)$. (The reason the log-likelihoods are lower for the last two specifications even though they contain more terms, is that the comparison is in terms of $\sum_{t \in \tau} \ln f\left(\epsilon_{t} ; \widehat{\sigma}_{t}\right)$ rather than the $\log$-likelihood of the ARMA-X representation, i.e. $\sum_{t \in \tau} \ln f\left(\widehat{u}_{t} ; \widehat{\sigma}_{u}\right)$, where $f$ is the normal density.) Also, the standard errors are high relative to the parameter estimates, so approximate $t$-tests with nulls $\lambda_{2}=0, \lambda_{3}=0$ and $\lambda_{4}=0$ do not reject at usual significance levels. All in all, then, this simple analysis does not suggest past volume nor past zeros or zero probabilities have an effect on volatility. 


\section{Conclusions}

We propose an estimation procedure for log-GARCH models that is asymptotically unbiased in the presence of zero returns, as long as there are not too many zeros. The algorithm relies on estimation via the ARMA representation, so it is not available for the Standard QMLE of Francq et al. (2013). The estimation procedure we propose distinguishes between true and observed return, and rests on the assumption that the true return is equal to zero with zero probability. This is compatible with observed return being zero due to missing values, non-trading, certain types of rounding or discreteness approximation error, impulse dummies in the mean specification to neutralise jumps or "outliers" and other data issues. In our framework zeros may occur non-randomly, randomly, or due to a combination of both. The zero probability can be time-varying, and volatility can depend on past zeros and zero probabilities. However, our framework is not guaranteed to be valid if the occurrence of a zero depends contemporaneously on the value of the de-volatilised return. Monte Carlo simulations show that volatility is generally underestimated when zeros are present if our algorithm is not used, and that our algorithm corrects the bias with the empirical standard errors corresponding well to their asymptotic counterparts. The empirical illustrations confirm that volatility is generally underestimated when zeros are present, and that the impact of shocks on volatility is underestimated in the presence of zeros. In practice this means the fitted conditional standard deviations are generally underestimated - sometimes substantially. Moreover, even when the average difference between risk-estimates is small, the day-to-day differences can be very large. Finally, a case study of Apple return, whose zero-probability has been steadily decreasing, suggests volatility does not depend on past zeros, nor on past zero-probabilities or the level of volume.

The results in this paper may be extended in several ways. First, if zeros are the result of measurement error of a true return whose zero probability is zero, then this also leads to biased estimates for other ARCH models, e.g. the GARCH of Bollerslev (1986), the EGARCH of Nelson (1991) and the Beta-t-EGARCH model of Harvey (2013). Estimation procedures in combination with an algorithm similar to the one we have proposed here can be used in all three classes. Second, the ideas in this paper can be used to develop an estimator in which the zero probability is truly non-zero, by appropriately scaling the return. This is pursued in Sucarrat and Grønneberg (2016).

\section{References}

Bollerslev, T. (1986). Generalized autoregressive conditional heteroscedasticity. Journal of Econometrics $31,307-327$.

Bondon, P. and N. Bahamonde (2012). Least squares estimation of ARCH models with missing observations. Journal of Time Series Analysis 33, 880-891.

Breidt, F. J. and A. L. Carriquiry (1996). Improved Quasi-Maximum Likelihood Estimation for Stochastic Volatility Models. In J. Lee, W. Johnson, and A. Zellner (Eds.), Modeling and Prediction: Honoring Seymour Geisser. Berlin: Springer. 
Brockwell, P. J. and R. A. Davis (2006). Time Series: Theory and Methods. New York: Springer. 2nd. Edition, first published in 1991.

Dempster, A., N. Laird, and D. Rubin (1977). Maximum likelihood estimation from incomplete data via the EM algorithm. Journal of the Royal Statistical Society Series B 39, 1-38.

Dickey, D. and W. Fuller (1979). Distribution of the estimators for autoregressive time series with a unit root. Journal of the American Statistical Association 74, 426-431.

Engle, R. (1982). Autoregressive Conditional Heteroscedasticity with Estimates of the Variance of United Kingdom Inflations. Econometrica 50, 987-1008.

Engle, R. F. and T. Bollerslev (1986). Modelling the persistence of conditional variances. Econometric Reviews 5, 1-50.

Francq, C., R. Roy, and A. Saidi (2011). Aasymptotic Properties of Weighted Least Squares Estimation in Weak PARMA Models. Journal of Time Series Analysis 32, 699-723.

Francq, C. and G. Sucarrat (2013). An Exponential Chi-Squared QMLE for Log-GARCH Models Via the ARMA Representation. http://mpra.ub.uni-muenchen.de/51783/.

Francq, C., O. Wintenberger, and J.-M. Zakoïan (2013). GARCH Models Without Positivity Constraints: Exponential or Log-GARCH? Journal of Econometrics 177, 34-36.

Francq, C. and J.-M. Zakoïan (2006). Linear-representation Based Estimation of Stochastic Volatility Models. Scandinavian Journal of Statistics 33, 785-806.

Francq, C. and J.-M. Zakoïan (2010). GARCH Models. New York: Marcel Dekker.

Geweke, J. (1986). Modelling the Persistence of Conditional Variance: A Comment. Econometric Reviews 5, 57-61.

Gomez, V. and A. Maravall (1994). Estimation, Prediction, and Interpolation for Nonstationary Series with the Kalman Filter. Journal of the American Statistical Association 89, 611-624.

Hannan, E. (1973). The asymptotic theory of linear time-series models. Journal of Applied Probability 10, $130-145$.

Harvey, A. C. (2013). Dynamic Models for Volatility and Heavy Tails. New York: Cambridge University Press.

Jones, R. (1980). Maximum likelihood fitting of ARMA models to time series with missing observations. Technometrics 22, 389-395.

Kohn, R. and C. Ansley (1986). Estimation, Prediction, and Interpolation for ARIMA Models With Missing Data. Journal of the American Statistical Association 81, 751-761.

Little, R. J. and D. B. Rubin (2002). Statistical Analysis with Missing Data. Hoboken, NJ: John Wiley and Sons. 2nd. Edition.

Ljung, G. and G. Box (1979). On a Measure of Lack of Fit in Time Series Models. Biometrika 66, $265-270$.

Milhøj, A. (1987). A Multiplicative Parametrization of ARCH Models. Research Report 101, University of Copenhagen: Institute of Statistics.

Nelson, D. B. (1991). Conditional Heteroskedasticity in Asset Returns: A New Approach. Econometrica $59,347-370$. 
Pantula, S. (1986). Modelling the Persistence of Conditional Variance: A Comment. Econometric Reviews 5, 71-73.

Psaradakis, Z. and E. Tzavalis (1999). On regression-based tests for persistence in logarithmic volatility models. Econometric Reviews 18, 441-448.

R Core Team (2015). R: A Language and Environment for Statistical Computing. Vienna, Austria: R Foundation for Statistical Computing.

Schwarz, G. (1978). Estimating the Dimension of a Model. The Annals of Statistics 6, 461-464.

Shumway, R. and D. Stoffer (1982). An approach to time series smoothing and forecasting using the EM-algorithm. Journal of Time Series Analysis 3, 254-264.

Sucarrat, G. (2014). lgarch: Simulation and estimation of log-GARCH models. http://cran.r-project . org/web/packages/lgarch/.

Sucarrat, G. and S. Grønneberg (2016). Models of Financial Return With Time-Varying Zero Probability. MPRA Paper No. 68931. Online at https://mpra.ub.uni-muenchen.de/68931/.

Sucarrat, G., S. Grønneberg, and Á. Escribano (2016). Estimation and Inference in Univariate and Multivariate Log-GARCH-X Models When the Conditional Density is Unknown. Computational Statistics and Data Analysis 100, 582-594.

Teräsvirta, T. (2009). An introduction to univariate GARCH models. In T. Mikosch, T. Kreiss, J.-P. Davis, R. Andersen, and T. Gustav (Eds.), Handbook of Financial Time Series. Berlin: Springer.

Wincek, M. A. and G. C. Reinsel (1986). An Exact Maximum Likelihood Estimation Procedure for Regression-ARMA Time Series Models with Possibly Nonconsecutive Data. Journal of the Royal Statistical Society B 48, 303-313.

Wintenberger, O. (2013). Continuous Invertibility and Stable QML Estimation of the EGARCH(1,1) model. Scandinavian Journal of Statistics 40, 846-867. 
Table 1: The Standard QMLE and the Gaussian ARMA-QMLE when zero returns are replaced by $c>0$ (see Section 3.1)

\begin{tabular}{|c|c|c|c|c|c|c|c|c|c|c|c|c|c|c|}
\hline \multirow[b]{3}{*}{$N(0,1):$} & \multirow{3}{*}{$\begin{array}{l}\text { DGP } \\
\left.\text { (ID: } \alpha_{0}, \alpha_{1}, \beta_{1}\right) \\
\mathrm{A}: 0,0.10,0.80\end{array}$} & \multirow[b]{2}{*}{$\pi_{0}$} & \multicolumn{6}{|c|}{ Standard QMLE: } & \multicolumn{6}{|c|}{ Gaussian ARMA-QMLE: } \\
\hline & & & $m\left(\hat{\alpha}_{0}\right)$ & $\operatorname{se}\left(\hat{\alpha}_{0}\right)$ & $m\left(\hat{\alpha}_{1}\right)$ & $\operatorname{se}\left(\hat{\alpha}_{1}\right)$ & $m\left(\beta_{1}\right)$ & $\operatorname{se}\left(\beta_{1}\right)$ & $m\left(\hat{\alpha}_{0}\right)$ & $\operatorname{se}\left(\hat{\alpha}_{0}\right)$ & $m\left(\hat{\alpha}_{1}\right)$ & $\operatorname{se}\left(\hat{\alpha}_{1}\right)$ & $m\left(\dot{\beta_{1}}\right)$ & $\operatorname{se}\left(\beta_{1}\right)$ \\
\hline & & 0.00 & -0.001 & 0.009 & 0.100 & 0.004 & 0.799 & 0.011 & -0.003 & 0.015 & 0.100 & 0.007 & 0.798 & 0.017 \\
\hline & & 0.05 & -0.020 & 0.010 & 0.097 & 0.005 & 0.807 & 0.012 & -0.019 & 0.018 & 0.071 & 0.007 & 0.826 & 0.021 \\
\hline & & 0.10 & -0.040 & 0.012 & 0.094 & 0.005 & 0.813 & 0.013 & -0.033 & 0.023 & 0.055 & 0.007 & 0.843 & 0.025 \\
\hline & & 0.20 & -0.076 & 0.015 & 0.089 & 0.005 & 0.826 & 0.013 & -0.195 & 0.579 & 0.034 & 0.010 & 0.767 & 0.403 \\
\hline & B: $0,0.05,0.90$ & 0.00 & -0.001 & 0.006 & 0.050 & 0.003 & 0.899 & 0.008 & -0.001 & 0.010 & 0.050 & 0.005 & 0.899 & 0.012 \\
\hline & & 0.05 & -0.011 & 0.007 & 0.048 & 0.004 & 0.903 & 0.009 & -0.012 & 0.014 & 0.036 & 0.005 & 0.912 & 0.016 \\
\hline & & 0.10 & -0.020 & 0.008 & 0.047 & 0.004 & 0.907 & 0.009 & -0.035 & 0.182 & 0.028 & 0.005 & 0.908 & 0.140 \\
\hline & & 0.20 & -0.039 & 0.011 & 0.044 & 0.004 & 0.913 & 0.010 & -0.225 & 0.662 & 0.018 & 0.007 & 0.797 & 0.450 \\
\hline & C: $0,0.03,0.95$ & 0.00 & -0.001 & 0.005 & 0.030 & 0.002 & 0.949 & 0.005 & -0.002 & 0.008 & 0.030 & 0.004 & 0.949 & 0.007 \\
\hline & & 0.05 & -0.007 & 0.006 & 0.028 & 0.002 & 0.952 & 0.005 & -0.007 & 0.010 & 0.023 & 0.004 & 0.955 & 0.009 \\
\hline & & 0.10 & -0.013 & 0.007 & 0.027 & 0.003 & 0.955 & 0.005 & -0.014 & 0.117 & 0.019 & 0.004 & 0.958 & 0.061 \\
\hline & & 0.20 & -0.024 & 0.009 & 0.025 & 0.003 & 0.959 & 0.006 & -0.138 & 0.600 & 0.013 & 0.005 & 0.906 & 0.286 \\
\hline \multirow{10}{*}{$t(5):$} & A: $0,0.10,0.80$ & 0.00 & -0.002 & 0.019 & 0.100 & 0.008 & 0.798 & 0.019 & -0.002 & 0.018 & 0.100 & 0.007 & 0.798 & 0.017 \\
\hline & & 0.05 & -0.026 & 0.021 & 0.095 & 0.007 & 0.807 & 0.018 & -0.021 & 0.022 & 0.076 & 0.007 & 0.822 & 0.020 \\
\hline & & 0.10 & -0.048 & 0.024 & 0.091 & 0.008 & 0.815 & 0.021 & -0.038 & 0.026 & 0.061 & 0.007 & 0.836 & 0.023 \\
\hline & & 0.20 & -0.091 & 0.029 & 0.084 & 0.008 & 0.828 & 0.023 & -0.080 & 0.156 & 0.042 & 0.007 & 0.847 & 0.093 \\
\hline & B: $0,0.05,0.90$ & 0.00 & -0.001 & 0.015 & 0.050 & 0.006 & 0.899 & 0.015 & -0.002 & 0.013 & 0.050 & 0.005 & 0.898 & 0.013 \\
\hline & & 0.05 & -0.015 & 0.01 & 0.048 & 0.006 & 0.90 & 0.0 & -0.0 & 0.01 & & & 0.910 & 0.014 \\
\hline & C: $0,0.03,0.95$ & 0.00 & -0.001 & 0.012 & 0.030 & 0.004 & 0.949 & 0.009 & -0.002 & 0.010 & 0.030 & 0.004 & 0.949 & 0.007 \\
\hline & & 0.05 & -0.010 & 0.01 & 0.02 & 0.004 & 0.953 & 0.0 & -0.007 & 0.012 & 0.024 & 0.004 & 0.954 & 0.008 \\
\hline & & 0.10 & -0.018 & 0.015 & 0.026 & 0.004 & 0.955 & 0.009 & -0.016 & 0.140 & 0.021 & 0.004 & 0.956 & 0.059 \\
\hline & & 0.20 & -0.031 & 0.020 & 0.023 & 0.004 & 0.959 & 0.011 & -0.175 & 0.795 & 0.015 & 0.005 & 0.901 & 0.314 \\
\hline
\end{tabular}

parameter identifier (i.e. A, B or C). $\pi_{0}$, the zero probability associated with $I_{t} \sim I I D, I_{t} \in\{0,1\}$. Standard QMLE, zeros replaced with $c=1$. Gaussian ARMA-QMLE, zeros replaced with $c=0.01 . m(\cdot)$, sample average of estimates. $s e(\cdot)$, sample standard deviation of estimates (division by $S$, not by $S-1$, where $S=1000$ is the number of Monte Carlo simulations). All computations in $R$, see R Core Team (2015). Simulations and estimation with the lgarch package version 0.5, see Sucarrat (2014). 
Table 2: The Gaussian ARMA-QMLE w/algorithm in the presence of zeros (see Section 3.2)

\begin{tabular}{|c|c|c|c|c|c|c|c|c|c|c|c|c|c|}
\hline & $\begin{array}{c}\text { DGP } \\
\left.\text { (ID: } \alpha_{0}, \alpha_{1}, \beta_{1}\right)\end{array}$ & $\pi_{0}$ & $m\left(\hat{\alpha}_{0}\right)$ & $s e\left(\hat{\alpha}_{0}\right)$ & $m\left(\hat{\alpha}_{1}\right)$ & $\operatorname{se}\left(\hat{\alpha}_{1}\right)$ & $\operatorname{ase}\left(\hat{\alpha}_{1}\right)$ & $m\left(\hat{\beta}_{1}\right)$ & $\operatorname{se}\left(\hat{\beta}_{1}\right)$ & $a \operatorname{se}\left(\hat{\beta}_{1}\right)$ & $m\left(\hat{E}\left(\ln z_{t}^{2}\right)\right.$ & $\operatorname{se}\left(\hat{E}\left(\ln z_{t}^{2}\right)\right.$ & $\operatorname{ase}\left(\hat{E}\left(\ln z_{t}^{2}\right)\right.$ \\
\hline \multirow[t]{12}{*}{$N(0,1):$} & A: $0,0.10,0.80$ & 0.00 & -0.003 & 0.015 & 0.100 & 0.007 & 0.007 & 0.798 & 0.017 & 0.017 & -1.270 & 0.018 & 0.017 \\
\hline & & 0.05 & 0.000 & 0.014 & 0.101 & 0.007 & 0.007 & 0.797 & 0.017 & 0.017 & -1.274 & 0.017 & 0.018 \\
\hline & & 0.10 & 0.003 & 0.015 & 0.102 & 0.008 & 0.007 & 0.796 & 0.018 & 0.018 & -1.277 & 0.018 & 0.018 \\
\hline & & 0.20 & 0.007 & 0.015 & 0.105 & 0.008 & 0.008 & 0.794 & 0.020 & 0.019 & -1.286 & 0.019 & 0.019 \\
\hline & B: $0,0.05,0.90$ & 0.00 & -0.001 & 0.010 & 0.050 & 0.005 & 0.005 & 0.899 & 0.012 & 0.013 & -1.270 & 0.017 & 0.017 \\
\hline & $D: 0,0.00,0.30$ & 0.05 & -0.001 & 0.011 & 0.050 & 0.006 & 0.005 & 0.898 & 0.014 & 0.013 & -1.272 & 0.017 & 0.018 \\
\hline & & 0.10 & 0.000 & 0.011 & 0.051 & 0.006 & 0.005 & 0.898 & 0.015 & 0.013 & -1.272 & 0.019 & 0.018 \\
\hline & & 0.20 & 0.003 & 0.011 & 0.052 & 0.006 & 0.006 & 0.897 & 0.015 & 0.014 & -1.277 & 0.020 & 0.019 \\
\hline & $\mathrm{C}: 0,0.03,0.95$ & 0.00 & -0.002 & 0.008 & 0.030 & 0.004 & 0.004 & 0.949 & 0.007 & 0.007 & -1.270 & 0.017 & 0.017 \\
\hline & & 0.05 & -0.002 & 0.009 & 0.031 & 0.004 & 0.004 & 0.948 & 0.008 & 0.007 & -1.272 & 0.018 & 0.018 \\
\hline & & 0.10 & -0.001 & 0.009 & 0.031 & 0.004 & 0.004 & 0.948 & 0.008 & 0.008 & -1.273 & 0.018 & 0.018 \\
\hline & & 0.20 & 0.000 & 0.009 & 0.032 & 0.005 & 0.004 & 0.947 & 0.009 & 0.008 & -1.275 & 0.019 & 0.019 \\
\hline \multirow[t]{12}{*}{$t(5):$} & A: $0,0.10,0.80$ & 0.00 & -0.002 & 0.018 & 0.100 & 0.007 & 0.007 & 0.798 & 0.017 & 0.017 & -1.568 & 0.027 & 0.028 \\
\hline & & 0.05 & 0.000 & 0.019 & 0.101 & 0.007 & 0.007 & 0.797 & 0.018 & 0.017 & -1.572 & 0.030 & 0.028 \\
\hline & & 0.10 & 0.002 & 0.019 & 0.103 & 0.008 & 0.007 & 0.795 & 0.018 & 0.018 & -1.575 & 0.028 & 0.029 \\
\hline & & 0.20 & 0.009 & 0.019 & 0.105 & 0.009 & 0.008 & 0.795 & 0.020 & 0.019 & -1.582 & 0.031 & 0.031 \\
\hline & B: $0,0.05,0.90$ & 0.00 & -0.002 & 0.013 & 0.050 & 0.005 & 0.005 & 0.898 & 0.013 & 0.013 & -1.568 & 0.027 & 0.028 \\
\hline & D. $0,0.00,0.30$ & 0.05 & -0.001 & 0.013 & 0.051 & 0.005 & 0.005 & 0.897 & 0.014 & & -1.568 & 0.033 & 0.028 \\
\hline & & 0.10 & $\begin{array}{c}-0.001 \\
0.000\end{array}$ & 0.013 & $\begin{array}{l}0.051 \\
0.051\end{array}$ & 0.006 & 0.005 & 0.897 & $\begin{array}{l}0.014 \\
0.014\end{array}$ & 0.013 & $\begin{array}{l}-1.000 \\
-1.571\end{array}$ & $\begin{array}{l}0.029 \\
0.029\end{array}$ & $\begin{array}{l}0.028 \\
0.029\end{array}$ \\
\hline & & 0.20 & 0.001 & 0.014 & 0.052 & 0.006 & 0.006 & 0.896 & 0.015 & 0.014 & -1.575 & 0.029 & 0.031 \\
\hline & C: $0,0.03,0.95$ & 0.00 & -0.002 & 0.010 & 0.030 & 0.004 & 0.004 & 0.949 & 0.007 & 0.007 & -1.567 & 0.027 & 0.028 \\
\hline & $0.0,0.00,0.50$ & 0.05 & -0.002 & 0.010 & 0.030 & 0.004 & 0.004 & 0.949 & 0.008 & 0.007 & -1.569 & 0.029 & 0.028 \\
\hline & & 0.10 & -0.002 & 0.010 & 0.031 & 0.004 & 0.004 & 0.948 & 0.008 & 0.008 & -1.569 & 0.029 & 0.029 \\
\hline & & 0.20 & 0.000 & 0.011 & 0.032 & 0.004 & 0.004 & 0.947 & 0.008 & 0.008 & -1.574 & 0.031 & 0.031 \\
\hline \multicolumn{14}{|c|}{ Panel $2\left(h_{t}=1.9+3.4 \cdot(t / T)\right):$} \\
\hline & (ID: ${ }_{\left.\alpha_{0}, \alpha_{1}, \beta_{1}\right)}^{\text {) }}$ & $\hat{\pi}_{0}$ & $m\left(\hat{\alpha}_{0}\right)$ & $\operatorname{se}\left(\hat{\alpha}_{0}\right)$ & $m\left(\hat{\alpha}_{1}\right)$ & $\operatorname{se}\left(\hat{\alpha}_{1}\right)$ & $\operatorname{ase}\left(\hat{\alpha}_{1}\right)$ & $m\left(\hat{\beta}_{1}\right)$ & $\operatorname{se}\left(\hat{\beta}_{1}\right)$ & ase $\left(\hat{\beta}_{1}\right)$ & $m\left(\hat{E}\left(\ln z_{t}^{2}\right)\right.$ & $\operatorname{se}\left(\hat{E}\left(\ln z_{t}^{2}\right)\right.$ & $\operatorname{ase}\left(\hat{E}\left(\ln z_{t}^{2}\right)\right.$ \\
\hline \multirow[t]{3}{*}{$N(0,1):$} & A: $0,0.10,0.80$ & 0.040 & 0.000 & 0.014 & 0.101 & 0.007 & 0.007 & 0.797 & 0.017 & 0.017 & -1.274 & 0.018 & 0.017 \\
\hline & B: $0,0.05,0.90$ & 0.039 & -0.001 & 0.010 & 0.050 & 0.005 & 0.005 & 0.898 & 0.013 & 0.013 & -1.272 & 0.017 & 0.017 \\
\hline & C: $0,0.03,0.95$ & 0.039 & -0.002 & 0.009 & 0.030 & 0.004 & 0.004 & 0.949 & 0.008 & 0.007 & -1.270 & 0.017 & 0.017 \\
\hline \multirow[t]{3}{*}{$t(5):$} & A: $0,0.10,0.80$ & & 0.000 & 0.018 & 0.101 & 0.007 & 0.007 & 0.798 & 0.017 & 0.017 & -1.569 & 0.031 & 0.028 \\
\hline & $\begin{array}{l}\text { A: } 0,0.10,0.80 \\
\text { B: } 0,0.05,0.90\end{array}$ & $\begin{array}{l}0.040 \\
0.040\end{array}$ & $\begin{array}{c}0.000 \\
-0.002\end{array}$ & $\begin{array}{l}0.018 \\
0.013\end{array}$ & $\begin{array}{l}0.101 \\
0.051\end{array}$ & $\begin{array}{l}0.007 \\
0.005\end{array}$ & 0.005 & $\begin{array}{l}0.798 \\
0.898\end{array}$ & 0.013 & 0.013 & $\begin{array}{l}-1.569 \\
-1.569\end{array}$ & $\begin{array}{l}0.031 \\
0.029\end{array}$ & $\begin{array}{l}0.028 \\
0.028\end{array}$ \\
\hline & C: $0,0.03,0.95$ & 0.040 & -0.002 & 0.011 & 0.031 & 0.004 & 0.004 & 0.948 & 0.008 & 0.007 & -1.567 & 0.028 & 0.028 \\
\hline \multicolumn{14}{|c|}{$\overline{\mathrm{DGP}}, \ln \sigma_{t}^{2}=\alpha_{0}+\alpha_{1} \ln \epsilon_{t-1}^{2}+\beta_{1} \ln \sigma_{t-1}^{2}$ with $z_{t} \sim I I D(0,1)$ and $T=10000 . N(0,1), z_{t}$ is standard normal with $E\left(\ln z_{t}^{2}\right) \approx-1.27$ and $\operatorname{Var}\left(z_{t}^{2}-\ln z_{t}^{2}\right) \approx 2.93 . t(5)$} \\
\hline \multicolumn{14}{|c|}{$z_{t}$ is standardised $t$ with 5 degrees of freedom, and with $E\left(\ln z_{t}^{2}\right) \approx-1.57$ and $\operatorname{Var}\left(z_{t}^{2}-\ln z_{t}^{2}\right) \approx 7.63$. ID, parameter identifier $\left(i . e\right.$. A, B or C). In Panel 2, $\hat{\pi}_{0}$ is the } \\
\hline
\end{tabular}


Table 3: Descriptive statistics and $\log$-GARCH estimates of six daily financial returns (see Section 4.1)

Descriptive statistics:

\begin{tabular}{llcccccc}
\hline & Sample & $s^{4}$ & $A R C H_{1}\left(r_{t}^{2}\right)$ & $p$-val & $T$ & $0 \mathrm{~s}$ & $\widehat{\pi}_{0}$ \\
\hline Apple & $10 / 9 / 1984-23 / 8 / 2013$ & 55.03 & 7.11 & 0.01 & 7303 & 294 & 0.040 \\
Ekornes & $4 / 1 / 2000-26 / 8 / 2013$ & 10.32 & 54.00 & 0.00 & 3546 & 667 & 0.188 \\
EUR/USD & $5 / 1 / 1999-18 / 1 / 2012$ & 5.44 & 139.39 & 0.00 & 3343 & 27 & 0.008 \\
SP500 & $5 / 1 / 1999-23 / 8 / 2013$ & 10.30 & 143.07 & 0.00 & 3684 & 2 & 0.001 \\
Oil & $5 / 4 / 1983-19 / 8 / 2013$ & 18.80 & 160.58 & 0.00 & 7621 & 73 & 0.010 \\
Gold & $4 / 1 / 2006-23 / 8 / 2013$ & 7.29 & 10.94 & 0.00 & 1929 & 20 & 0.010 \\
\hline
\end{tabular}

$\log -\operatorname{GARCH}(1,1)$ :

\begin{tabular}{lcccccccc}
\hline & & $\widehat{\alpha}_{0}$ & $\widehat{\alpha}_{1}$ & $s e\left(\widehat{\alpha}_{1}\right)$ & $\widehat{\beta}_{1}$ & $s e\left(\widehat{\beta}_{1}\right)$ & $A R C H_{2}$ & $p$-val \\
\hline Apple & 0-adj & 0.034 & 0.014 & 0.003 & 0.983 & 0.005 & 64.7 & 0.00 \\
& Algo & 0.048 & 0.029 & 0.004 & 0.967 & 0.005 & 19.2 & 0.00 \\
Ekornes & 0-adj & 0.374 & 0.087 & 0.016 & 0.784 & 0.059 & 42.1 & 0.00 \\
& Algo & 0.074 & 0.047 & 0.011 & 0.943 & 0.016 & 15.6 & 0.00 \\
EUR/USD & 0-adj & 0.024 & 0.020 & 0.004 & 0.973 & 0.006 & 7.3 & 0.03 \\
& Algo & 0.025 & 0.022 & 0.005 & 0.971 & 0.007 & 7.9 & 0.02 \\
SP500 & 0-adj & 0.070 & 0.045 & 0.006 & 0.946 & 0.008 & 61.8 & 0.00 \\
& Algo & 0.071 & 0.046 & 0.006 & 0.946 & 0.008 & 59.4 & 0.00 \\
Oil & 0-adj & 0.074 & 0.043 & 0.004 & 0.951 & 0.005 & 87.0 & 0.00 \\
\multirow{3}{*}{ Gold } & Algo & 0.075 & 0.046 & 0.004 & 0.948 & 0.005 & 76.6 & 0.00 \\
& 0-adj & 0.055 & 0.029 & 0.006 & 0.959 & 0.009 & 0.8 & 0.68 \\
& Algo & 0.058 & 0.033 & 0.006 & 0.956 & 0.009 & 0.6 & 0.75 \\
\hline
\end{tabular}

$\log -\operatorname{GARCH}(1,1) \mathrm{w} /$ asymmetry:

\begin{tabular}{lcccccccccc}
\hline & & $\widehat{\alpha}_{0}$ & $\widehat{\alpha}_{1}$ & $s e\left(\widehat{\alpha}_{1}\right)$ & $\widehat{\beta}_{1}$ & $s e\left(\widehat{\beta}_{1}\right)$ & $\widehat{\lambda}_{1}$ & $s e\left(\widehat{\lambda}_{1}\right)$ & $A R C H_{2}\left(\widehat{z}_{t}^{2}\right)$ & $p$-val \\
\hline Apple & 0 -adj & 0.042 & 0.015 & 0.004 & 0.978 & 0.008 & 0.007 & 0.005 & 47.1 & 0.00 \\
& Algo & 0.047 & 0.016 & 0.005 & 0.968 & 0.005 & 0.023 & 0.006 & 21.0 & 0.00 \\
Ekornes & 0-adj & 0.340 & 0.066 & 0.017 & 0.793 & 0.057 & 0.063 & 0.025 & 31.4 & 0.00 \\
& Algo & 0.034 & 0.007 & 0.008 & 0.973 & 0.009 & 0.042 & 0.009 & 15.0 & 0.00 \\
\multirow{4}{*}{ SPR500 } & 0-adj & 0.023 & 0.024 & 0.005 & 0.973 & 0.006 & -0.008 & 0.006 & 4.6 & 0.10 \\
& Algo & 0.023 & 0.026 & 0.006 & 0.971 & 0.007 & -0.010 & 0.006 & 4.6 & 0.10 \\
Oil & 0-adj & 0.068 & 0.037 & 0.007 & 0.948 & 0.008 & 0.015 & 0.009 & 59.1 & 0.00 \\
& Algo & 0.069 & 0.039 & 0.007 & 0.947 & 0.008 & 0.013 & 0.009 & 57.4 & 0.00 \\
Gold & 0 -adj & 0.072 & 0.032 & 0.005 & 0.952 & 0.005 & 0.021 & 0.007 & 80.2 & 0.00 \\
& Algo & 0.072 & 0.035 & 0.005 & 0.950 & 0.005 & 0.019 & 0.007 & 77.9 & 0.00 \\
& 0-adj & 0.055 & 0.028 & 0.008 & 0.959 & 0.009 & 0.004 & 0.012 & 0.8 & 0.68 \\
& Algo & 0.058 & 0.036 & 0.008 & 0.956 & 0.009 & -0.006 & 0.012 & 0.5 & 0.77 \\
\hline
\end{tabular}

$s^{4}$, sample kurtosis. $A R C H_{k}(x)$ and $p$-val, Ljung and Box (1979) test statistic for $k$ th. order autocorrelation in $x$ and its $p$-value. $T$, number of returns. 0 s, number of zero returns. $\widehat{\pi}_{0}$, proportion of zero returns. 0-adj, zero returns replaced by the minimum of the absolute non-zero value before estimation. Algo, estimation with algorithm (i.e. zeros not replaced, but treated as missing values). se (.), standard error of estimate (based on the numerically estimated Hessian of the ARMA representation). All computations in $R$, see R Core Team (2015). Estimation with the lgarch package version 0.5 (Sucarrat (2014)) using the Gaussian ARMA-QMLE. 
Table 4: Descriptive statistics of fitted conditional standard deviations (see Section 4.1)

\begin{tabular}{|c|c|c|c|c|c|c|c|c|}
\hline & \multicolumn{4}{|c|}{ Apple: } & \multicolumn{4}{|c|}{ SP500: } \\
\hline & Mean & $s^{2}$ & Max & Min & Mean & $s^{2}$ & Max & Min \\
\hline 0-adj & 2.97 & 0.75 & 6.35 & 1.51 & 1.19 & 0.33 & 4.73 & 0.44 \\
\hline Algo & 2.99 & 0.80 & 6.17 & 1.10 & 1.19 & 0.33 & 4.77 & 0.43 \\
\hline Diff & 0.01 & 0.36 & 1.90 & -1.09 & 0.00 & 0.00 & 0.39 & -0.01 \\
\hline \multirow[t]{3}{*}{ Ratio } & 1.02 & 0.06 & 2.14 & 0.63 & 1.00 & 0.00 & 1.36 & 0.99 \\
\hline & \multicolumn{4}{|c|}{ Ekornes: } & \multicolumn{4}{|c|}{ Oil: } \\
\hline & Mean & $s^{2}$ & Max & Min & Mean & $s^{2}$ & Max & Min \\
\hline 0 -adj & 2.36 & 0.22 & 5.28 & 1.30 & 2.20 & 0.97 & 7.53 & 0.41 \\
\hline Algo & 2.57 & 0.39 & 5.47 & 1.35 & 2.19 & 0.90 & 7.42 & 0.43 \\
\hline Diff & 0.21 & 0.27 & 3.23 & -0.93 & -0.01 & 0.02 & 1.04 & -0.22 \\
\hline \multirow[t]{3}{*}{ Ratio } & 1.10 & 0.06 & 2.61 & 0.66 & 1.01 & 0.01 & 1.66 & 0.94 \\
\hline & \multicolumn{4}{|c|}{ EUR/USD: } & \multicolumn{4}{|c|}{ Gold: } \\
\hline & Mean & $s^{2}$ & Max & Min & Mean & $s^{2}$ & Max & Min \\
\hline 0-adj & 0.66 & 0.02 & 1.19 & 0.36 & 1.32 & 0.10 & 2.58 & 0.72 \\
\hline Algo & 0.66 & 0.02 & 1.19 & 0.37 & 1.33 & 0.12 & 2.88 & 0.67 \\
\hline Diff & 0.00 & 0.00 & 0.08 & -0.03 & 0.01 & 0.01 & 0.48 & -0.07 \\
\hline Ratio & 1.00 & 0.00 & 1.16 & 0.95 & 1.00 & 0.00 & 1.26 & 0.92 \\
\hline
\end{tabular}

Mean, sample average. $s^{2}$, sample variance. Max, maximum value. Min, minimum value. Diff, the difference between fitted conditional standard deviations: $\widehat{\sigma}_{t, \text { Algo }}-\widehat{\sigma}_{t, 0 \text {-adj }}$. Ratio, the ratio between fitted conditional standard deviations: $\widehat{\sigma}_{t, \text { Algo }} / \widehat{\sigma}_{t, 0 \text {-adj }}$. All computations in $R$, see R Core Team (2015).

Table 5: Models of the zero probability of daily Apple returns (see Section 4.2)

\begin{tabular}{lccccccc}
\hline \hline Model & $\widehat{\rho}_{0}$ & $s e\left(\widehat{\rho}_{0}\right)$ & $\widehat{\rho}_{1}$ & $s e\left(\widehat{\rho}_{1}\right)$ & $k$ & $\log L$ & BIC \\
\hline$h_{t}=\rho_{0}$ & 3.171 & 0.060 & - & - & 1 & -1232.465 & 0.3387 \\
$h_{t}=\rho_{0}+\rho_{1} \cdot(t / T)$ & 1.870 & 0.094 & 3.437 & 0.263 & 2 & -1123.887 & 0.3102 \\
$h_{t}=\rho_{0}+\rho_{1} \ln V_{t}$ & -16.081 & 1.374 & 1.212 & 0.088 & 2 & -1122.887 & 0.3100 \\
$h_{t}=\rho_{0}+\rho_{1} \ln P_{t}$ & 1.230 & 0.156 & 0.808 & 0.072 & 2 & -1134.604 & 0.3132
\end{tabular}

Dynamic logit models where $\pi_{1 t}=1 /\left(1+\exp \left(-h_{t}\right)\right), h_{t}=\ln \left(\pi_{1 t} / \pi_{0 t}\right), V_{t}$ is the traded volume in USD and $P_{t}$ is the stock price. $s e(\cdot)$, standard error of estimate (computed as the square root of the diagonal of $-\widehat{H}^{-1}$, where $\widehat{H}$ is the numerically estimated Hessian). Estimation by maximum likelihood. $k$, number of parameters. $\log L$, the attained $\log$-likelihood. BIC, the Schwarz (1978) information criterion computed in terms of the average $\log$-likelihood $\log L / T$. All computations in $R$, see R Core Team (2015). 
Table 6: Models of Apple stock return volatility (see Section 4.2)

\begin{tabular}{|c|c|c|c|c|c|c|c|c|c|}
\hline Model & $\widehat{\alpha}_{0}$ & $\begin{array}{c}\widehat{\alpha}_{1} \\
(s . e .)\end{array}$ & $\begin{array}{c}\widehat{\beta}_{1} \\
(\text { s.e. })\end{array}$ & $\begin{array}{c}\hat{\lambda}_{1} \\
(s . e .)\end{array}$ & $\begin{array}{l}\widehat{\lambda}_{2} \\
(\text { s.e. })\end{array}$ & $\begin{array}{c}\hat{\lambda}_{3} \\
(s . e .)\end{array}$ & $\begin{array}{l}\hat{\lambda}_{4} \\
(s . e .)\end{array}$ & $\log L^{*}$ & $\mathrm{BIC}$ \\
\hline 1 & 2.266 & - & - & - & - & - & - & -17885.217 & 5.105 \\
\hline 2 & 0.047 & $\begin{array}{l}0.016 \\
(0.005)\end{array}$ & $\begin{array}{l}0.968 \\
(0.005)\end{array}$ & $\begin{array}{l}0.023 \\
(0.006)\end{array}$ & - & - & - & -17237.252 & 4.924 \\
\hline 3 & 0.080 & $\begin{array}{l}0.017 \\
(0.005)\end{array}$ & $\begin{array}{l}0.967 \\
(0.005)\end{array}$ & $\begin{array}{l}0.023 \\
(0.006)\end{array}$ & $\begin{array}{c}-0.002 \\
(0.001)\end{array}$ & - & - & -17243.879 & 4.927 \\
\hline 4 & 5.723 & $\begin{array}{l}0.017 \\
(0.005)\end{array}$ & $\begin{array}{l}0.967 \\
(0.006) \\
\end{array}$ & $\begin{array}{l}0.023 \\
(0.006) \\
\end{array}$ & $\begin{array}{r}-0.428 \\
(0.782) \\
\end{array}$ & $\begin{array}{l}0.011 \\
(0.029) \\
\end{array}$ & $\begin{array}{l}0.351 \\
(0.601) \\
\end{array}$ & -17242.810 & 4.929 \\
\hline $\begin{array}{l}\text { Gaussian } \\
\ln \sigma_{t}^{2}= \\
\text { error of } \\
\text { tion). Lo } \\
\widehat{\sigma}_{t} \text { is the } \\
\text { Schwarz } \\
\log L^{*} / T \\
(2015)) \mathrm{w}\end{array}$ & $\begin{array}{l}\text { ARM } \\
\alpha_{0}+c \\
\text { stimate } \\
g L^{*}, \log \\
\text { fitted co } \\
(1978)\end{array}$ & $\begin{array}{l}\text { A-QML } \\
1 \text { ln } \epsilon_{t-1}^{2} \\
\text { (based } \\
\text {-likeliho } \\
\text { nditiona } \\
\text { informat }\end{array}$ & $\begin{array}{r}\text { estim } \\
+\beta \ln c\end{array}$ & $\begin{array}{l}{ }_{t-1}^{2}+ \\
\text { umerica } \\
\text { uted as } \\
\text { d devia } \\
\text { rion }\end{array}$ & $\begin{array}{l}\text { algorithn } \\
\ln V_{t-1} \\
\text { y estima } \\
\sum_{t \in \tau} \ln f \\
\text { ion and } \\
\text { nputed }\end{array}$ & $\begin{array}{c}\text { of } \\
\lambda_{2} I_{t-1} \\
\text { ed Hess }\end{array}$ & $\begin{array}{r}\text { pecifica } \\
+\lambda_{3} \widehat{h} \\
\text { an of } t\end{array}$ & $\begin{array}{l}\text { tions } \\
-1 \cdot \quad s e(\cdot), \\
\text { he ARMA re }\end{array}$ & $\begin{array}{l}\text { ned in } \\
\text { tandarc } \\
\text { resenta- } \\
\text { density } \\
\text { is. BIC } \\
\text { relihooc } \\
\text { ce Team }\end{array}$ \\
\hline
\end{tabular}



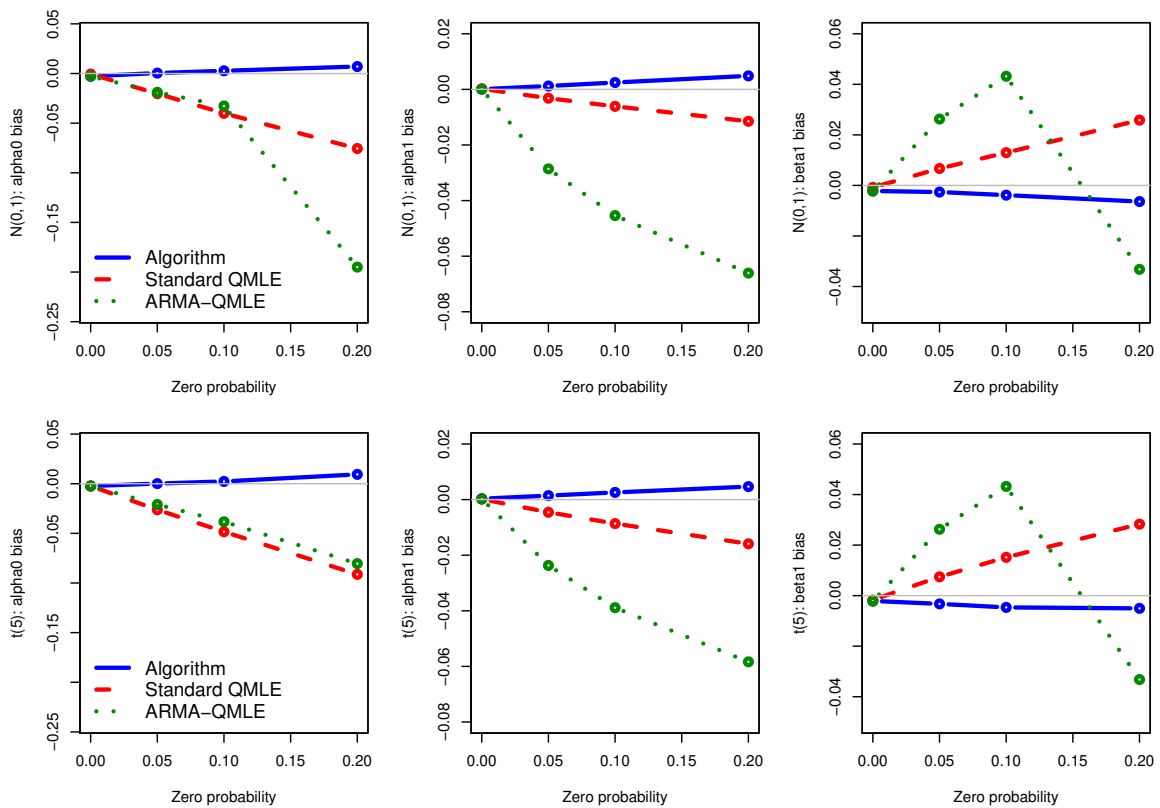

Figure 1: Bias (estimate - true value) of three estimators for parameter combination A, see Sections 3.1 and 3.2
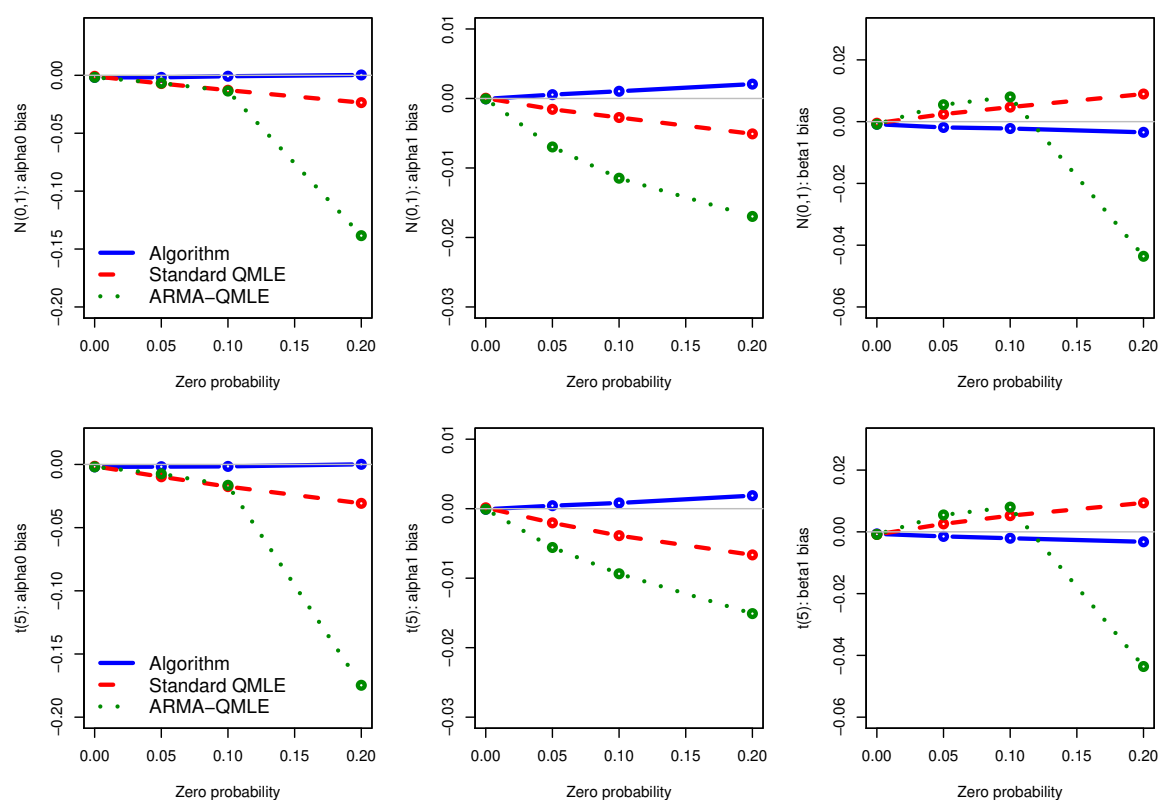

Figure 2: Bias (estimate - true value) of three estimators for parameter combination C, see Sections 3.1 and 3.2 

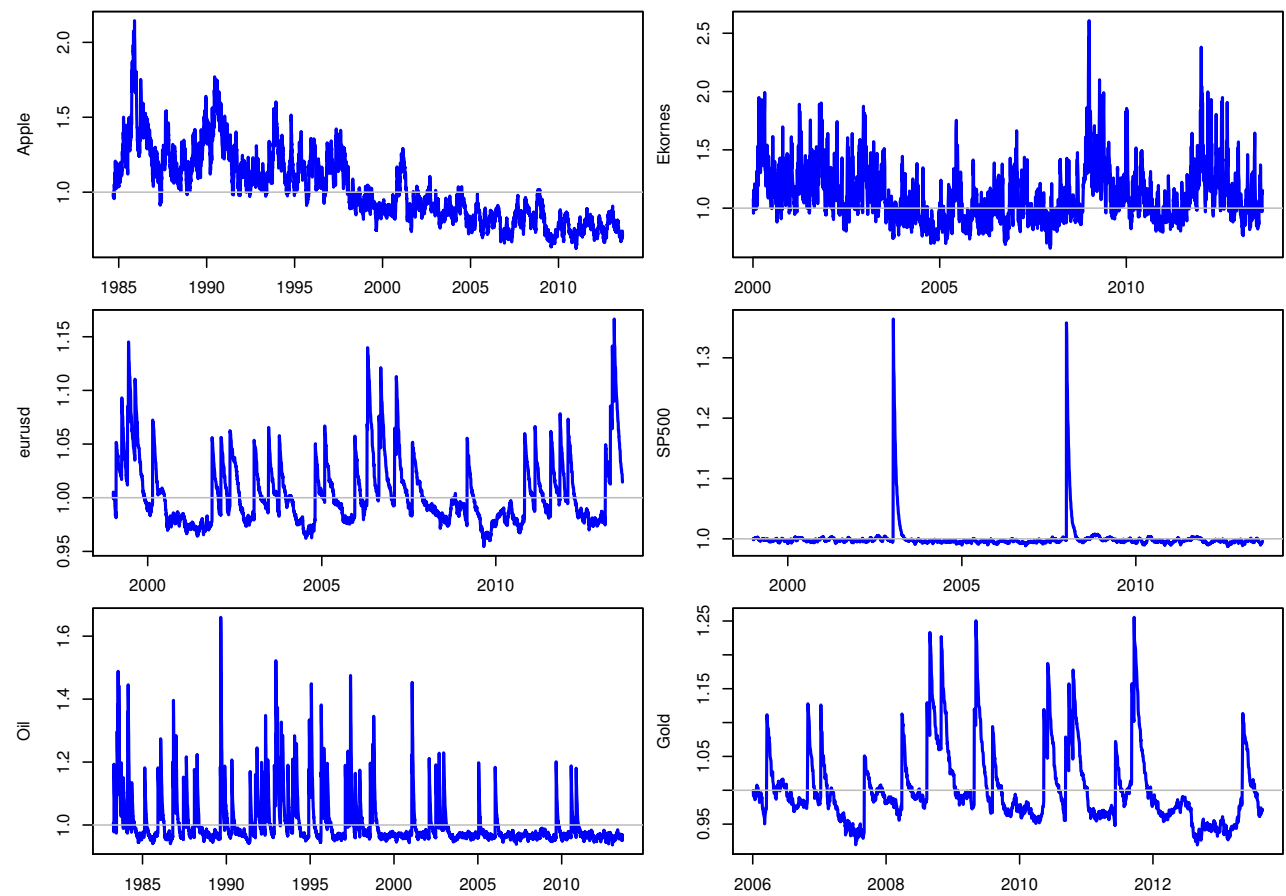

Figure 3: Ratios of fitted conditional standard deviations (i.e. $\left.\widehat{\sigma}_{t, \mathrm{Alg} o} / \widehat{\sigma}_{t, 0 \text {-adj }}\right)$, see Section 4.1

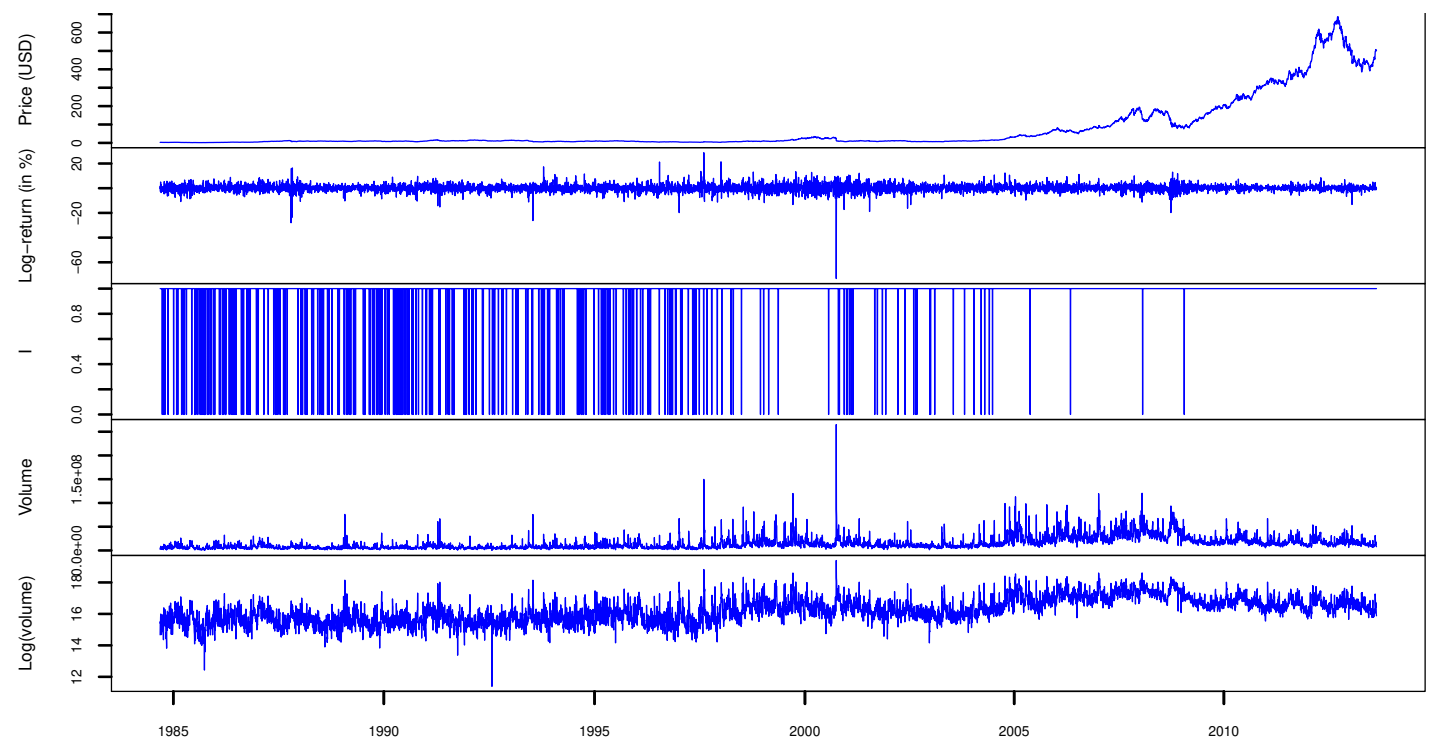

Figure 4: The Apple stock price (in USD), observed return, $I_{t}$, volume and log-volume from 10 September 1984 to 23 August 2013 ( $T=7303$ observations), see Section 4.2 